\title{
Surfactants role on the deformation of colliding small bubbles
}

\author{
D.S. Valkovska, K.D. Danov*, I.B. Ivanov \\ Laboratory of Thermodynamics and Physico-Chemical Hydrodynamics, Faculty of Chemistry, University of Sofia, \\ 1164 Sofia, Bulgaria
}

Received 29 December 1998; accepted 10 March 1999

\begin{abstract}
The mutual approach of two bubbles and the rate of thinning and the deformation of the partially mobile thin liquid film intervening between them is studied. The material properties of the interfaces (surface viscosity, Gibbs elasticity, surface and/or bulk diffusivity) are taken into account. In the normal stress balance at the fluid interfaces we include the contribution of the intermolecular forces. To obtain the liquid velocity and pressure distribution the lubrication approximation is used. From the normal stress boundary condition the first order (with respect to the capillary number) shape function is derived. It provides information on the inversion thickness, at which the curvature in the gap between the drops changes from convex to concave, and the pimple thickness, at which the curvature of the interfaces spontaneously increases due to the action of the attractive intermolecular forces. The analytical and numerical investigations reveal significant influence of the disjoining pressure and the surfactant on both thicknesses. Explanation of the following effects is proposed: (i) increase of the pimple thickness and decrease of the inversion thickness with the increase of the interfacial mobility; (ii) role of the surface viscosity; (iii) role of the van der Waals interaction. (C) 1999 Elsevier Science B.V. All rights reserved.
\end{abstract}

Keywords: Influence of surfactant; Inversion thickness; Mobility of interfaces; Pimple thickness; Small bubbles; Velocity of approach

\section{Introduction}

The collision of two foam bubbles may be accompanied by deformation (flattening in the zone of contact), which is governed by the hydrodynamic resistance and the energy of interaction between the bubbles. When the bubbles (or drops) approach each other, the slight deformation of their surfaces at large separations can increase

\footnotetext{
* Corresponding author.
}

significantly as they come closer to each other and at a given thickness the interfacial shape can change from convex to concave: a dimple is formed. The conditions for flattening in the case of different driving forces (buoyancy, Brownian, etc. forces) are investigated in Refs. [1-3]. Due to the instability of the dimple it flows out soon after the formation and an almost plane-parallel film is formed $[4,5]$. This film thins to the final critical thickness of rupture without significantly changing its radius [6]. The thickness, at which the 
dimple forms was called earlier [4,5] inversion thickness, $h_{\mathrm{it}}$, because the surface curvature changes sign. Since the dimple expands radially very fast and almost immediately a planar film between the bubbles forms [6], it seems appropriate to call $h_{\text {it }}$ also film formation thickness.

This physical picture takes place if the drops are relatively large (of the order of $1 \mathrm{~mm}$ ). On the contrary, due to the high capillary pressure, small drops keep their shape practically spherical up to the moment of flocculation or coalescence. Since the coalescence rate depends strongly on whether a film forms between the bubbles or they remain (almost) spherical up to the moment of coalescence, for many chemical technologies [7,8] it is important to be able to predict, at least qualitatively, the occurrence of this process. Which pathway the bubbles will follow depends on many factors: their size; surface tension; surfactant type and concentration; surface interaction; external forces; etc. This makes the computational study of the process extremely difficult and actually impossible for systems of practical interest. That is why we tried to develop an analytical approach. Although our results can be hardly applied directly to practical systems, we believe that our conclusions can serve as 'thumb rules' to help practitioners in their own studies. Our results are also applicable to emulsion drops, provided that the surfactant is dissolved in the continuous phase, which is usually the case with practical systems then the liquid circulation in the drops has no effect on the rate of mutual approach and from hydrodynamic viewpoint the drops behave as bubbles $[4,5]$.

The first solution of the problem for two rigid spherical particles, approaching each other along their line of centers in a liquid is due to Taylor [9]. For a long time the Taylor formula for the velocity of approach was used to calculate the flocculation rate not only of suspensions, but also of emulsions. In the case of two spherical drops, when the surfactant is soluble only in the continuous phase, simple asymptotic expression was derived by Ivanov et al. [10]. It takes into account the influence of Gibbs elasticity, bulk and surface diffusivities, but the effects of the interfacial viscosity and the disjoining pressure were not consid- ered. Numerical calculations of the film profile during the approach of two droplets in pure liquid phases were performed by Davis et al. [11,12]. These authors derived also some asymptotic expressions. Yiantsios and Davis [13] studied numerically the deformation of two approaching small droplets in pure liquid phases (no surfactant present) under the action of an external driving force and van der Waals attraction force. Similarly to Ivanov et al. $[5,10]$, they found two different regimes: without dimple formation and with dimple formation. They showed also that at a given gap width, $h_{\mathrm{pt}}$, the van der Waals attraction can prevail over the hydrodynamic resistance and observed at even smaller thickness increase of the curvature in the gap region (a pimple appears). The thickness $h_{\mathrm{pt}}$ is called below the pimple thickness.

In this paper we extend the previous studies of bubbles approach by deriving analytical expressions for the bubble shape and approach rate, based on an asymptotic procedure. We study the role of the interaction forces between the drop surfaces and of the surfactant (more specifically of Gibbs elasticity and surface viscosity). In Section 2 a mathematical model, based on the lubrication approximation, is formulated in order to derive the governing equations for the surface velocity, the pressure and the local thickness. It is followed in Section 3 by a study of the different patterns of bubbles deformation (the problem for inversion and pimple thicknesses). Several asymptotic expressions, accounting for the Gibbs elasticity, the surface viscosity, the driving force, and the van der Waals disjoining pressure on the approach velocity and film deformation are derived in Section 4 . The general solution is briefly discussed in Section 5 .

\section{Mathematical formulation of the problem}

We consider a thin viscous liquid layer between two gas bubbles, 1 and 2, which is squeezed by the external force, $F$ (Fig. 1). To simplify the discussion we will assume that the bubbles radii are the same. However, all obtained results can be applied to bubbles with different radii as well, 
provided that the surface velocity $U$ and the bubble radius $R_{\mathrm{c}}$ in all equations are replaced by the mean values $U=\left(U_{1}+U_{2}\right) / 2$ and $R_{\mathrm{c}}=2 R_{\mathrm{c}, 1} R_{\mathrm{c}, 2} /$ $\left(R_{\mathrm{c}, 1}+R_{\mathrm{c}, 2}\right)$ of the surface velocities $U_{1}$ and $U_{2}$ and radii, $R_{\mathrm{c}, 1}$ and $R_{\mathrm{c}, 2}$, of the two bubbles (see Fig. 1). The problem is described in a cylindrical coordinate system, Orz, and the local thickness of the layer is denoted by $H(r, t)$. Since the bubbles are supposed to be close to each other, the hydrodynamic resistance is concentrated in the gap region, where the radii of curvature are large compared to the local distance between the interfaces. Therefore the lubrication approximation can be used for solution of the problem $[10,14,15]$. The main requirement for applicability of this approximation are: (i) small Reynolds number (the inertia terms in the momentum balance equation can be neglected); (ii) small thickness of the viscous layer between the bubbles compared to the characteristic bubble radii; (iii) small slope of the interfaces. In the lubrication approximation the pressure, $P$, in the continuous phase depends only on the radial coordinate, $r$, and time, $t$ : $P=P(r, t)$. Then the integration of the equations of fluid motion and continuity, with account for the corresponding kinematic boundary conditions (continuity of velocities), leads to the integrated

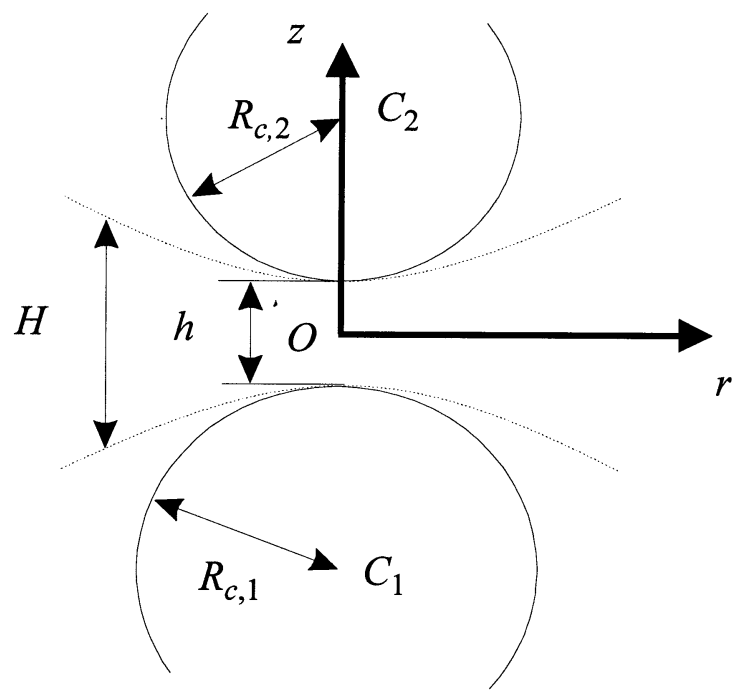

Fig. 1. Sketch of two approaching bubbles of radii $R_{\mathrm{c}, 1}$ and $R_{\mathrm{c}, 2}$ at a distance $h$. bulk continuity equation (see Eq. (3d) in Ref. [10] and Eqs. (2, 3) in Ref. [14])

$-V+\frac{1}{r} \frac{\partial}{\partial r}\left[r H\left(U-\frac{H^{2}}{12 \eta} \frac{\partial P}{\partial r}\right)\right]=0$

In Eq. (1) $\eta$ is the dynamic viscosity, $V(r, t)=-$ $\partial H / \partial t$ is the local velocity of film thinning, and $U$ is the radial component of the surface velocity.

The surfactant distribution can be found by solving the diffusion equation along with the surfactant balance at the film surfaces and the following assumptions: (iv) small Peclet number in the gap (the convective terms can be neglected); (v) small deviation from equilibrium of the subsurface concentration and adsorption (the perturbations $\delta c$ and $\delta \Gamma$ of the equilibrium subsurface concentration, $c_{0}$, and adsorption, $\Gamma_{0}$, are so small, that the surface material parameters are constant during the drainage); (vi) the surfactants are soluble only in the continuous phase (in this case the viscous friction from the fluid in the droplets is negligible compared to the friction from the continuous phase-the emulsion system behaves as a foam, see Eq. (25) and the discussions below it in Ref. [4]); (vii) Diffusion-controlled adsorption (the surfactant exchange between the bulk fluid and the interface is diffusion controlled which is usually the case with thin films $[4,5])$. The result of this solution gives the dependence of the adsorption, $\Gamma$, on the surface velocity, $U$, and on the local film thickness, $H$. Then, by means of the tangential stress balance boundary condition (which accounts for the role of the surface tension gradient and the surface viscosity - the Marangoni and Boussinesq effects, respectively) one obtains (see Eq. (12) in Ref. [14])

$\frac{H}{12 \eta R_{\mathrm{c}}} \frac{\partial P}{\partial r}=-\frac{U}{R_{\mathrm{c}}\left(h_{\mathrm{s}}+b H\right)}+N_{\mathrm{sv}} \frac{\partial}{\partial r}\left[\frac{1}{r} \frac{\partial}{\partial r}(r U)\right]$

In Eq. (2) $R_{\mathrm{c}}$ is the bubble radius, $b$ and $h_{\mathrm{s}}$ are the bulk and surface diffusion parameters, and $N_{\mathrm{sv}}$ is the dimensionless surface viscosity number. These parameters are defined as:

$b \equiv \frac{3 \eta D}{h_{\mathrm{a}} E_{\mathrm{G}}}, \quad h_{\mathrm{s}} \equiv \frac{6 \eta D_{\mathrm{s}}}{E_{\mathrm{G}}}, \quad N_{\mathrm{sv}} \equiv \frac{\eta_{\mathrm{s}}}{6 \eta R_{\mathrm{c}}}$ 
where $E_{\mathrm{G}} \equiv-(\partial \sigma / \partial \ln \Gamma)_{0}$ and $h_{\mathrm{a}} \equiv(\partial \Gamma / \partial c)_{0}$ are the equilibrium values of the Gibbs elasticity and the adsorption length, $\sigma$ is the surface tension, $D$ and $D_{\mathrm{s}}$ are the bulk and surface diffusion coefficients, and the sum of the interfacial shear, $\eta_{\mathrm{sh}}$, and dilatational, $\eta_{\text {dil }}$, viscosities, is the total surface viscosity, $\eta_{s} \equiv \eta_{\mathrm{sh}}+\eta_{\mathrm{dil}}$. The parameters defined by Eq. (3) do not depend on the thickness. They can vary in a wide range depending on the type of surfactants and the surfactant concentration (for more information see Section 5).

The layer profile is determined from the normal stress balance at the interfaces which in lubrication approximation reduces to balance of pressures $[5,10]$ :

$P_{\mathrm{m}}+\frac{2 \sigma}{R_{\mathrm{c}}}=P+\Pi+\frac{\sigma}{2 r} \frac{\partial}{\partial r}\left(r \frac{\partial H}{\partial r}\right)$

where $P_{\mathrm{m}}$ is the pressure in the spherical meniscus at infinite distance from the axis of symmetry and $\Pi$ is the disjoining pressure.

Finally, an integral force balance is needed to close the problem:

$F=2 \pi \int_{0}^{\infty}\left(P-P_{\mathrm{m}}\right) r \mathrm{~d} r+W$,

$W \equiv 2 \pi \int_{0}^{\infty} \Pi r \mathrm{~d} r$

where $W$ is the force due to the action of the disjoining pressure, П. Eq. (5) relates the external force, $F$, acting on the approaching bubbles (droplets) and the drag (lubrication) force - the latter is the integral in Eq. (5), which depends only weakly on time [13]. The character of the external force, $F$, depends on the process under consideration, e.g. buoyancy force for the process of creaming [16], turbulence force in a stirred emulsion [17] or Brownian force in a fine emulsion at rest [1]. It is important to note that in the framework of the assumptions (i-vi) it is not possible to make a transition to zero surfactant concentration. In such case the full low Reynolds hydrodynamic model has to be used [11-13] or the next order terms with respect to Reynolds and Peclet numbers in the lubrication approximation equation have to be included [5].

Let $h$ be the minimum distance between the caps of the undeformed bubbles. We will suppose that $h \ll R_{\mathrm{c}}$. Then, the appropriate lengthscales inside the thin film (the narrow region around the axis of symmetry) in the $z$ - and $r$-directions are $h$ and $\sqrt{h R_{\mathrm{c}}}$, respectively [10]. It was shown by Taylor [9] that the approach rate of two particles (with tangentially immobile surfaces) at such close separation obeys the equation:

$V_{\mathrm{Ta}}=\frac{2 h F}{3 \pi \eta R_{\mathrm{c}}^{2}}$

The quantity $V_{\text {Ta }}$, which we call Taylor velocity, is the appropriate scale for the rate of approach of the two bubbles. It follows then from the continuity equation and the normal stress boundary condition that the appropriate scales for the radial velocity, the dynamic and disjoining pressure, and the time are $V_{\mathrm{Ta}} \sqrt{R_{\mathrm{c}} / h}, 12 V_{\mathrm{Ta}} \eta R_{\mathrm{c}} / h$, and $h / V_{\mathrm{Ta}}$, respectively. Hence, we introduce the following dimensionless groups:

$$
\begin{aligned}
& r \equiv x \sqrt{h R_{\mathrm{c}}}, \quad t \equiv \frac{h}{V_{\mathrm{Ta}}} \tau, \quad H \equiv h \tilde{H}, \\
& U \equiv V_{\mathrm{Ta}} \tilde{U} \sqrt{\frac{R_{\mathrm{c}}}{h}}, \quad V \equiv V_{\mathrm{Ta}} \tilde{V} \\
& P-P_{\mathrm{m}} \equiv 12 V_{\mathrm{Ta}} \eta \frac{R_{\mathrm{c}}}{h^{2}} \tilde{P}, \quad \Pi \equiv 12 V_{\mathrm{Ta}} \eta \frac{R_{\mathrm{c}}}{h^{2}} \tilde{Q}, \\
& W \equiv 12 \pi V_{\mathrm{Ta}} \eta \frac{R_{\mathrm{c}}^{2}}{h} \tilde{W}, h_{s} \equiv h \tilde{h}_{s}, \alpha \equiv \frac{F}{2 \pi \sigma h}
\end{aligned}
$$

Here $x, \tau, \tilde{H}, \tilde{U}, \tilde{V}, \tilde{P}, \tilde{Q}$ and $\tilde{W}$ are the respective nondimensional values of the radial coordinate, $r$, time, $t$, the local thickness, $H$, the surface velocity, $U$, the local approaching velocity, $V$, the pressure difference, $P-P_{\mathrm{m}}$, the disjoining pressure, $\Pi$, and the force due to the action of the disjoining pressure, $W ; \tilde{h_{\mathrm{s}}}$ is the nondimensional parameter $h_{\mathrm{s}}$, which takes into account the influence of the surface diffusion, and the parameter $\alpha$ plays the same role as Bond's number in the theory of capillarity.

With the scaling introduced above, the dimensionless creeping flow Eqs. (1), (2), (4) and (5) inside the thin gap transform to:

$\frac{1}{x} \frac{\partial}{\partial x}\left[x \tilde{H}\left(\tilde{U}-\tilde{H}^{2} \frac{\partial \tilde{P}}{\partial x}\right)\right]=\tilde{V}$ 


$$
\begin{aligned}
& \tilde{H} \frac{\partial \tilde{P}}{\partial x}=-\frac{\tilde{U}}{b \tilde{H}+\tilde{h}_{\mathrm{s}}}+N_{\mathrm{sv}} \frac{\partial}{\partial x}\left[\frac{1}{x} \frac{\partial}{\partial x}(x \tilde{U})\right] \\
& 8 \alpha(\tilde{P}+\tilde{Q})=1-\frac{1}{4 x} \frac{\partial}{\partial x}\left(x \frac{\partial \tilde{H}}{\partial x}\right) \\
& 1=16 \int_{0}^{\infty} \tilde{P} x \mathrm{~d} x+8 \tilde{W}
\end{aligned}
$$

We will assume that the bubbles are only slightly deformed, which means that the dimensionless parameter $\alpha$ is small compared to the unity $(\alpha \ll 1)$. Then the local thickness, $\tilde{H}$, the pressure, $\tilde{P}$, the surface velocity, $\tilde{U}$, and the local velocity, $\tilde{V}$, can be expanded in series in terms of the power of $\alpha$ :

$$
\begin{gathered}
\tilde{H}=\tilde{H}_{0}+\alpha \tilde{H}_{1}+\ldots, \quad \tilde{P}=\tilde{P}_{0}+\alpha \tilde{P}_{1}+\ldots, \\
\tilde{U}=\tilde{U}_{0}+\alpha \tilde{U}_{1}+\ldots, \quad \tilde{V}=\tilde{V}_{0}+\alpha \tilde{V}_{1}+\ldots
\end{gathered}
$$

By substituting these series in Eqs. $(8 \mathrm{a}, 8 \mathrm{~d})$ and separating the terms with the same power of $\alpha$ one obtains series of simpler equations.

The shape of the colliding bubbles is determined mainly by the viscous stress, the capillary pressure and the disjoining pressure. Depending on the relative magnitude of these factors one can envisage at least two kinds of deformation:

\subsection{Formation of a dimple}

It is observed when there is no significant attractive (negative) disjoining pressure and the capillary pressure is not large enough to counteract the normal viscous stress and the positive component of the disjoining pressure. Then it may happen that at a given gap width (called inversion thickness, $h_{\mathrm{it}}$ [18]), the bubbles deform significantly and their caps become flat $[5,18]$. Since the flat surface cannot sustain the viscous stress, the interfacial shape in the gap changes suddenly from convex to concave, i.e. a dimple forms. The subsequent evolution of the dimple can follow different paths $[5,10,18]$ but at least with small bubbles the dimple is either pushed out or gradually decreases until a thin (almost) plane-parallel film forms between the bubbles. Since at the inversion thickness the surface is flat, its curvature must be zero, so that one has: $\frac{1}{x} \frac{\partial}{\partial x}\left(x \frac{\partial \tilde{H}}{\partial x}\right)=0 \quad$ at $\quad x=0$

or

$8 \alpha(\tilde{P}+\tilde{Q})=1 \quad$ at $\quad x=0$

The second expression above follows from Eq. (8c). This equation is nothing else but the condition for balance of the dynamic and disjoining pressure in the gap and the capillary pressure in the spherical parts of the bubbles (the latter corresponds to the unity).

\subsection{Formation of a pimple}

If the disjoining pressure, $\Pi$, is negative, the two film surfaces will attract each other, i.e. the disjoining pressure will counteract the hydrodynamic pressure thus decreasing the deformation. Because of the different dependence of these pressures on the gap width, $h$, (usually $\Pi$ depends stronger on $h$ ), it may happen that at a given distance, $h_{\mathrm{pt}}$, the disjoining pressure effect totally eliminates the viscous deformation of the caps of the bubbles (around the axis of symmetry), so that at this moment the capillary pressure there [the last term in the right hand side of Eq. (4)] is exactly $2 \sigma / R_{c}$. Then the sum of the dynamic pressure, $P-P_{\mathrm{m}}$, and the disjoining pressure, $\Pi$, becomes zero [see Eq. (4)]. Further decrease of the thickness, $h$, will make the sum $P-P_{\mathrm{m}}+\Pi$ negative. Although the drag force is an integral on the pressures over the drop area [see Eq. (5)], one can argue that at $h_{\mathrm{pt}}$ the drag force will be (approximately) zero. At smaller thicknesses it will become negative and the force balance (5) will stop being possible. Then protrusions will form at the drop caps, which will start growing spontaneously until coalescence occurs, unless there is another factor to prevent that. Because of its shape, we will call this protrusion (which is opposite to the dimple) 'pimple' and the thickness, $h_{\mathrm{pt}}$, at which it forms, 'pimple formation thickness'. The above discussion suggests that $h_{\mathrm{pt}}$ can be found from the condition [see Eq. (8c)]:

$\tilde{P}+\tilde{Q}=0 \quad$ at $\quad x=0$

The pimple formation can be interpreted as the onset of instability without fluctuations (stability 
analysis of the film intervening between the drops will be carried out in Ref. [19]). The drop deformation due to the negative disjoining pressure was first found numerically in Ref. [13] for the case of two drops in a system without surfactants.

\section{Tangentially immobile interfaces}

If the bulk solution contains large amount of surfactant (close to or above the critical micelle concentration, $\mathrm{CMC}$ ), the Gibbs elasticity, $E_{\mathrm{G}}$, may be high enough to suppress the interfacial motion and to make the interfaces tangentially immobile, i.e. then $\tilde{U}=0$ [4]. In this section we will use this simplification in Eqs. (8a, c, d).

As already discussed, we will seek the solution of the problem (8) as asymptotic series for $\tilde{H}$ and $\tilde{P}$ in terms of the powers of $\alpha$. Substituting the series from Eq. (9) into Eqs. (8a, c, d) and equalizing the coefficients in front of the same powers of $\alpha$, one obtains sets of simpler equations for $\tilde{H}_{0}$, $\tilde{P}_{0}$ and $\tilde{V}_{0}$ (zeroth order approximation); for $\tilde{H}_{1}$, $\tilde{P}_{1}$, and $\tilde{V}_{1}$ (first order approximation); etc. It is important to note that every following term in the series for the local thickness, $\tilde{H}_{\mathrm{n}}$, can be calculated from Eq. (8c) independently of $\tilde{P}_{\mathrm{n}}$ by using only the previous terms in the asymptotic expansion.

The zeroth order solutions for $\tilde{H}_{0}$ and $\tilde{P}_{0}$ are:

$\tilde{H}_{0}=1+x^{2}, \quad \tilde{P}_{0}=\frac{\tilde{V}_{0}}{8 \tilde{H}_{0}^{2}}$

After substituting the expression for $\tilde{P}_{0}$ from Eq. (12) into the zeroth order approximation of Eq.

(8d) one obtains the zero order term of the velocity of thinning:

$\tilde{V}_{0}=1-8 \tilde{W}_{0}, \quad \tilde{W}_{0}=2 \int_{0}^{\infty} \tilde{Q}_{0} x \mathrm{~d} x$

where $\tilde{Q}_{0}$ is the zero order term in the disjoining pressure and $\tilde{W}_{0}$ is the zeroth order term in the force due to the action of the disjoining pressure. Since the disjoining pressure, $\tilde{Q}$, and the energy of interaction, $\tilde{W}$, depend only on the local film thickness, $\tilde{H}$, their zeroth orders are determined only by $\tilde{H}_{0}$. The intermolecular forces of longest range are the van der Waals forces [20,21]. In the common case of small thicknesses (no electromag-

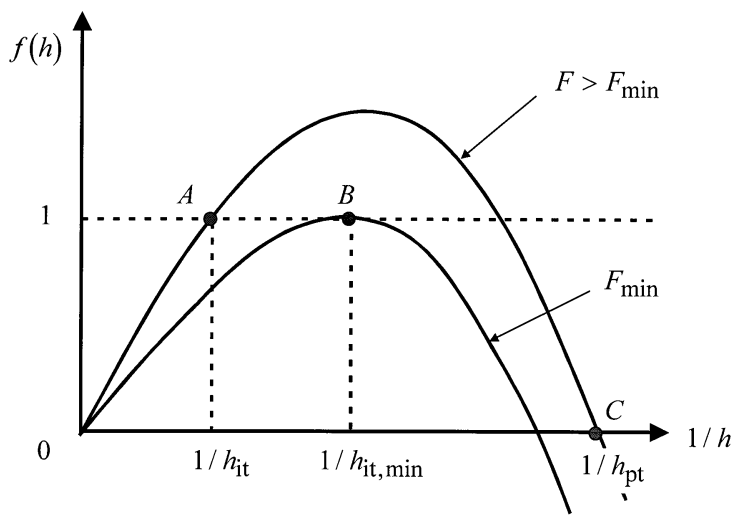

Fig. 2. Sketch of the dependence of function $f(h)$ on $1 / h$. The pimple thickness, $h_{\mathrm{pt}}$, is the solution of the equation $f\left(1 / h_{\mathrm{pt}}\right)=$ 0 (see point $C$ ). The inversion thickness, $h_{\mathrm{it}}$, is the smaller solution of the equation $f\left(1 / h_{\mathrm{it}}\right)=1$ (see point $A$ ). The case of minimal force, $F_{\min }$, is the point $B$.

netic retardation) the van der Waals disjoining pressure satisfies Derjaguin approximation [22] and has the following simple form:

$\tilde{Q}=-\frac{\tilde{A}}{4 \tilde{H}^{3}}, \quad \tilde{A} \equiv \frac{A_{\mathrm{H}}}{18 \pi \eta V_{\mathrm{Ta}} R_{\mathrm{c}} h}$

where $A_{\mathrm{H}}$ is the Hamaker constant and $\tilde{A}$ is its dimensionless form. From Eqs. (12)-(14) it follows that the zero order term of the velocity of thinning obey the simple relationship:

$\tilde{V}_{0}=1+\tilde{A}$

Therefore, the attractive van der Waals disjoining pressure accelerates the droplet approach compare to the classical Taylor regime. Finally, after substituting the solutions from Eqs. (12)-(15) into the equation for the inversion thickness [Eq. (10)] we obtain $\alpha(1-\tilde{A})=1$. With Eq. (7) and Eq. (14) this leads to the following equation for $h_{\mathrm{it}}$ :

$f\left(h_{\mathrm{it}}\right) \equiv \frac{F}{2 \pi \sigma h_{\mathrm{it}}}\left(1-\frac{A_{\mathrm{H}} R_{\mathrm{c}}}{12 F h_{\mathrm{it}}^{2}}\right)=1$

(for discussion of the validity of this equation see the end of this section). In the case when the intermolecular forces are negligible, from Eq. (16) the known result, $h_{\mathrm{it}}=F /(2 \pi \sigma)$, can be derived $[10,18]$.

As sketched in Fig. 2, the function $f\left(h_{\mathrm{it}}\right)$ in the left hand side of Eq. (16) has a maximum at $h_{\mathrm{it}}^{2}=A_{\mathrm{H}} R_{\mathrm{c}} /(4 F)$. From the two sets of possible 
solutions for the dimple formation thickness we select those from the left branch of the curve (point A in Fig. 2), since the right branch corresponds to smaller inversion thickness. In order for Eq. (16) to have a solution at all, its left hand side must reach values larger than 1 so that the condition for existence of the solution for the dimple (film) formation thickness will be:

$\frac{4 F^{3}}{9 \pi^{2} \sigma^{2} A_{\mathrm{H}} R_{\mathrm{c}}} \geq 1$

Thus, we reach the conclusion that in the presence of van der Waals attraction the dimple will form only with appropriate values [satisfying Eq. (17)] of the system parameters $F, A_{\mathrm{H}}, R_{c}$ and $\sigma$. If the other parameters are fixed, the driving force, $F$, must be larger than $F_{\min } \equiv\left(2.25 \pi^{2} \sigma^{2} R_{c} A_{\mathrm{H}}\right)^{1 / 3}$. If the driving force takes this value the minimum possible inversion thickness is (see point B in Fig. 2)

$$
h_{\mathrm{it}, \text { min }}=\frac{F_{\min }}{3 \pi \sigma}=\left(\frac{A_{\mathrm{H}} R_{\mathrm{c}}}{12 \pi \sigma}\right)^{1 / 3}
$$

Therefore, for tangentially immobile interfaces the van der Waals attraction causes a slight decrease of the inversion thickness, from $F /(2 \pi \sigma)$ to $F /$ $(3 \pi \sigma)$. The limitations for the other parameters can be obtained in a similar way from Eq. (17) it is obvious that large attractive forces and surface tensions will hinder and even suppress the dimple formation. The situation with the bubble radius is slightly more complicated, because $F$ also depends on $R_{\mathrm{c}}$, e.g. for gravity coalescence $F \propto R_{\mathrm{c}}^{3}$ so that large bubbles will form dimples much more easily. Substituting the solution from Eqs. (12)-(15) into Eq. (11) one derives for the pimple thickness ${ }^{1}$ :

$h_{\mathrm{pt}}=\sqrt{\frac{A_{\mathrm{H}} R_{\mathrm{c}}}{12 F}}$

\footnotetext{
${ }^{1}$ One of the Referees mentioned to us the recent paper of Cristini et al. [32]. These authors calculated numerically the influence of an insoluble nondiffusing surfactant on the velocity of approach of spherical drops. They took also into account the influence of the van der Waals attraction and found numerically that the rapid coalescence occurs at $h<h_{\mathrm{pt}}$ (see Eq. (6.1) in Ref. [32]).
}

This result shows that in the presence of van der Waals attraction the solution for the pimple thickness always exists. A comparison between Eq. (10) and Eq. (11) reveals that $h_{\mathrm{pt}}$ corresponds to the intersection point (point $\mathrm{C}$ in Fig. 2) of the curve $f(h)$ with the abscissa (the right hand side of Eq. (16) must be zero, rather than 1). Hence, when the solution for the inversion thickness exists, $h_{\mathrm{pt}}$ is always smaller than $h_{\mathrm{it}}$ and the pimple appearance is impossible, because it is preceded by dimple formation. In such case the only possible mechanism for film rupture is through fluctuational instability $[5,23]$.

Eq. (19) shows that the pimple formation thickness depends significantly on the drop radius, $R_{\mathrm{c}}$. For example, if a bubble is driven by buoyancy and is approaching another bubble (suppose immobile), the force is $F=4 \pi R_{\mathrm{c}}^{3} \Delta \rho \mathrm{g} / 3$, where $\Delta \rho$ is the density difference and $g$ is the gravity acceleration. Then $h_{\mathrm{pt}}^{2}=A_{\mathrm{H}} /\left(16 \pi \Delta \rho g R_{\mathrm{c}}^{2}\right)$, and it is inversely proportional to the bubble (drop) radius. For typical values of the Hamaker constant $A_{\mathrm{H}}=$ $4 \times 10^{-20} \mathrm{~J}$, density difference (for drops) $\Delta \rho=$ $0.12 \times 10^{3} \mathrm{~kg} \mathrm{~m}^{-3}$, and $R_{\mathrm{c}}=10 \mu \mathrm{m}$, the pimple thickness is $82.3 \mathrm{~nm}$. Note that this thickness is quite large, so that when calculating the van der Waals energy of interaction the electromagnetic retardation must be accounted for [21].

The above conclusions are illustrated in Fig. 3, showing the dependence of the inversion, $h_{\mathrm{it}}$, and the pimple, $h_{\mathrm{pt}}$, thicknesses on the drop radius, $R_{\mathrm{c}}$, for tangentially immobile surfaces. As physical parameters in our numerical investigation we used the experimental data from Refs. [24,25] for a system of two approaching small droplets of soybean oil in water. The aqueous film is stabilized by bovine serum albumin (BSA) with $0.15 \mathrm{M}$ sodium chloride to suppress the electrostatic interaction. The density difference, $\Delta \rho$, is $0.072 \times 10^{3}$ $\mathrm{kg} \mathrm{m}^{-3}$ and the experimentally determined interfacial tension, $\sigma$, was reported therein to be 7,15 and $30 \mathrm{mN} \mathrm{m}^{-1}$ for different concentrations of BSA. When calculating the van der Waals attraction at small and large distances, the Hamaker constant was replaced by the effective interaction parameter, $A_{\text {eff }}$, defined by Eqs. (5.9.1-5) in Ref. [21]. It takes into account the electromagnetic 
retardation. For large distances $A_{\text {eff }}$ is inversely proportional to the local film thickness and for small distances it is equal to the Hamaker constant, $A_{\mathrm{H}}$. One sees from Fig. 3 that the larger the bubble radius, the earlier the film forms, i.e. $h_{\mathrm{it}}$ is greater-it reaches $500 \mathrm{~nm}$ for drop radii of the order of $200 \mu \mathrm{m}$. The equation determining the inversion thickness [Eq. (16)] has no solution for drop radii smaller than $80 \mu \mathrm{m}$. The pimple thickness, $h_{\mathrm{pt}}$, for the same system is also shown on the figure. It decreases with the increase of the drop radius, i.e. with larger driving force.

Another question worthwhile discussing is the validity of Eq. (16). It is easy to see that with negligible intermolecular forces it is in fact equivalent to $\alpha=1$. This could cast some doubt on the results because, according to the initial assumption, $\alpha$ must be a small parameter. This apparent contradiction stems from the nature of the asymptotic procedure we used. Without entering into details we will note that the same problem could be solved by assuming that the dimensional local thickness, $H$, can be represented as superposition of the unperturbed value, $H_{0}=h+r^{2} /\left(2 R_{\mathrm{c}}\right)$, and perturbations $H_{1}, H_{2}$, etc. (such an approach was used in Refs. [11-13]). The perturbation must satisfy the conditions $H_{1} \ll H_{0}, H_{2} \ll H_{1}$, etc. In order to check if this condition is fulfilled by our solution, we calculated the next term in the expan- sion in Eq. (9). By using Eqs. (8c), (9) and (12) we thus obtained the following result for the dimensionless local thickness of the gap: ${ }_{\tilde{x}}^{2}$

$\tilde{H}=1+x^{2}-\alpha \ln \left(1+x^{2}\right)+\alpha \tilde{A} \frac{x^{2}}{1+x^{2}}+O\left(\alpha^{2}\right)$

If for simplicity we neglect the van der Waals interactions, we have

$\left|\frac{H_{1}}{H_{0}}\right|=\left|\frac{\alpha \tilde{H}_{1}}{\tilde{H}_{0}}\right|=\alpha \frac{\ln \left(1+x^{2}\right)}{1+x^{2}}$

The maximum value of the right hand side of Eq. (21) is $0.178 \alpha$ for $x<0.5$, which means that even for $\alpha=1$ the perturbation $\alpha \tilde{H}_{1}$ is smaller than the unperturbed gap width $\tilde{H}_{0}$. The logarithmic term in Eq. (20) can be derived also directly by integrating the normal stress condition [Eq. (8c)] and using the integrated force balance [Eq. (8d)].

\section{Effect of small and large surface viscosities}

In the case of low molecular weight surfactants the surface viscosity is usually negligible at low surfactant concentrations. On the contrary, the effect of the Gibbs elasticity (Marangoni effect), which also damps the surface mobility, is significant even at low surfactant concentrations. The surface viscosity may become sizable only when

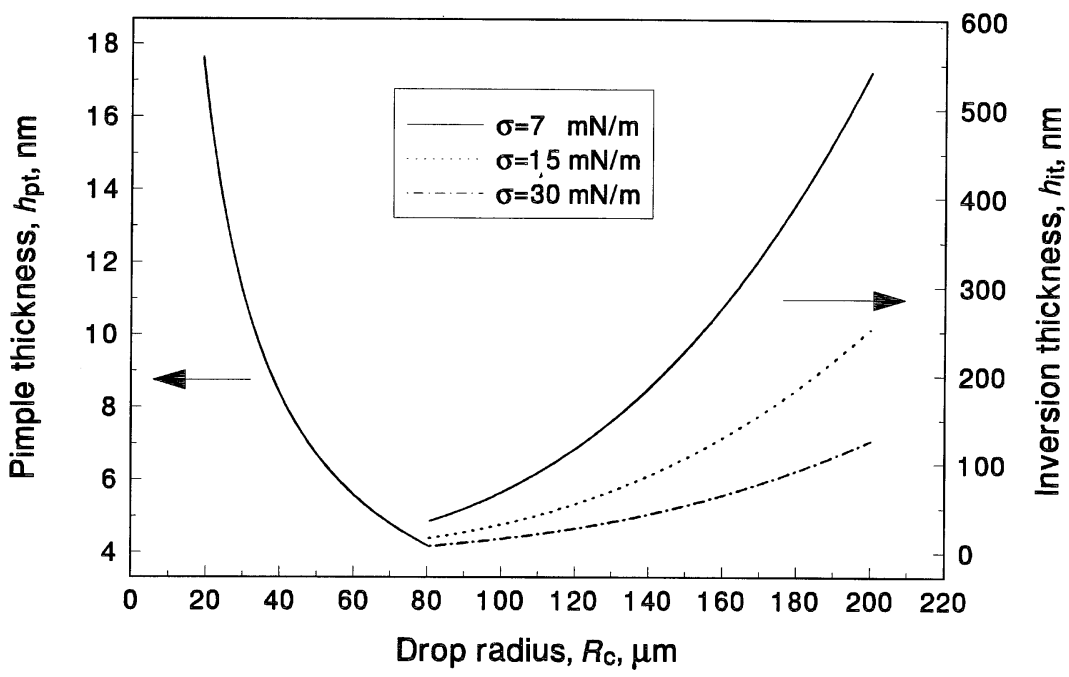

Fig. 3. Effect of the drop radius, $R_{\mathrm{c}}$, on the inversion, $h_{\mathrm{it}}$, and pimple, $h_{\mathrm{pt}}$, thickness for tangentially immobile surfaces. 
the surface coverage is (almost) complete (at adsorption $\left.\Gamma=\Gamma_{\infty}\right)$. However, at these surface concentrations the Gibbs elasticity is also very high - theoretically it must become infinity and the surface must become immobile (due only to the Marangoni effect) at $\Gamma=\Gamma_{\infty}$, if the surfactant obeys Langmuir adsorption isotherm [see Eq. (27) below]. Hence, the effect of the surface viscosity for low molecular surfactants will be always dominated by the effect of Gibbs elasticity-e.g. it was shown in Refs. $[4,5,14,15]$ that for a planar film with radius $100 \mu \mathrm{m}$ in order to have an effect comparable to that of the Gibbs elasticity, the surface viscosity must be larger than $1 \mathrm{sp}$. That is why we believe that the role of the surface viscosity in foam and emulsion stability has been somewhat overstated in the literature [26,27]. The situation can be different with proteins (or other polymers), which have relatively low Gibbs elasticity and high surface viscosities. The surface viscosity may prove important also in processes with small radial lengthscale, such as short surface waves or the initial stage of dimple formation or fluid motion around the dimple rim (which is very narrow) in the case of large and stable, persisting dimples [5] — indeed, it has been shown [4,5,14,15] that the relative contribution of the surface viscosity (compared with Gibbs elasticity) is inversely proportional to the square of the radial lengthscale. The effect of the surface viscosity is enhanced also by the surfactant bulk diffusion, which leads to decrease of the adsorption gradient thus decreasing the effect of the Gibbs elasticity. Since these issues have not yet been resolved and to demonstrate more clearly the competition between Gibbs elasticity and surface viscosity, we have studied briefly the effect of the latter on the dimple and pimple formation.

The parameters, which account for the effects of surface viscosity and Gibbs elasticity are: $N_{\mathrm{sv}} h_{\mathrm{s}} /$ $h=\eta_{\mathrm{s}} D_{\mathrm{s}} /\left(h E_{\mathrm{G}} R_{\mathrm{c}}\right)$ and $N_{\mathrm{sv}} b=\eta_{\mathrm{s}} D /\left(h_{\mathrm{a}} E_{\mathrm{G}} R_{\mathrm{c}}\right)$ (typical values of the parameters for three different type of surfactants are given in Section 5). In this section we will assume that both parameters are small and will solve the problem [Eqs. $(8 \mathrm{a}-\mathrm{d})]$ up to zeroth order terms. The surface velocity, $\tilde{U}_{0}$, and the velocity of bubbles' approach, $\tilde{V}_{0}$, are related with the pressure gradient, $\partial \tilde{P}_{0} / \partial x$, as

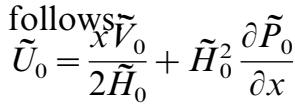

[this follows from Eq. (8a)]. If we substitute Eq. (22) into Eq. (8b), the corresponding dimensionless form of the tangential stress balance becomes

$$
-\frac{x \tilde{V}_{0}}{2 \tilde{H}_{0}^{2}}+\frac{(b+1) \tilde{H}_{0}+\tilde{h}_{\mathrm{s}}}{\tilde{H}_{0}\left(b \tilde{H}_{0}+\tilde{h}_{\mathrm{s}}\right)} \tilde{U}_{0}=N_{\mathrm{sv}} \frac{\partial}{\partial x}\left[\frac{1}{x} \frac{\partial}{\partial x}\left(x \tilde{U}_{0}\right)\right]
$$

Finally, for the velocity of bubbles approach, $V_{0}$, we obtain from Eqs. (8d), (22) and (23)

$V_{0}=V_{\mathrm{Ta}}\left[1-\frac{\pi R_{\mathrm{c}}}{F} W_{0}(h)\right] \Phi_{\mathrm{v}}\left(h, b, d, N_{\mathrm{sv}}\right)$

where $d$ is a length parameter defined as $d \equiv h_{\mathrm{s}}$ / $(b+1)$ [10]. From Eqs. (10) and (11) the respective transcendental equations for the inversion thickness, $h_{\mathrm{it}}$, and the pimple thickness, $h_{\mathrm{pt}}$, are:

$$
\begin{aligned}
& \frac{F}{2 \pi \sigma h_{\mathrm{it}}}\left[1-\frac{\pi R_{\mathrm{c}}}{F} W_{0}\left(h_{\mathrm{it}}\right)\right] \Phi_{\mathrm{p}}\left(h_{\mathrm{it}}, b, d, N_{\mathrm{sv}}\right) \\
& \quad+\frac{R_{\mathrm{c}}}{2 \sigma} \Pi\left(h_{\mathrm{it}}\right)=1 \\
& \frac{F}{\pi R_{\mathrm{c}} h_{\mathrm{pt}}}\left[1-\frac{\pi R_{\mathrm{c}}}{F} W_{0}\left(h_{\mathrm{pt}}\right)\right] \Phi_{\mathrm{p}}\left(h_{\mathrm{pt}}, b, d, N_{\mathrm{sv}}\right)+\Pi\left(h_{\mathrm{pt}}\right) \\
& \quad=0
\end{aligned}
$$

In Eqs. $(24,25 \mathrm{a}, \mathrm{b})$ the mobility functions, $\Phi_{\mathrm{v}}$ and $\Phi_{\mathrm{p}}$, take into account the influence of the interfacial rheological parameters on the velocity (see Appendix A). At high elasticity the length parameter, $d$, is much smaller than $h$, i.e. $d / h \approx$ $6 \eta D_{\mathrm{s}} /\left(E_{\mathrm{G}} h\right) \rightarrow 0$. Then we have $\Phi_{\mathrm{v}} \rightarrow 1$ and $\Phi_{\mathrm{p}} \rightarrow 1$ and Eqs. (24, 25a, b) reduce to the result for tangentially immobile interfaces (see Section 3). At small surfactant concentration, or when the film thickness is very small, $d / h \gg 1$ and $\Phi_{\mathrm{p}} \rightarrow 1 /$ $\ln (d / h)$. Hence, the smaller the surfactant concentration the lower the dynamic pressure in the gap, and the dimple appears at smaller distances while the pimple formation occurs at higher distances between the droplets. Briefly, decreasing the surfactant concentration favors formation of a pimple rather than of a dimple. 
Table 1

Dependence of the mobility functions, $\Phi_{\mathrm{v}}$ and $\Phi_{\mathrm{p}}$, on the surface rheological parameters and bulk diffusivity

\begin{tabular}{llll}
\hline No. & Particular cases & Mobility functions, $\Phi_{\mathrm{v}}$ and $\Phi_{\mathrm{p}}$ & \\
\hline Case 1 & $d / h \ll 1$ & $\Phi_{\mathrm{v}}=(b+1) /\left[1-\frac{d}{3 h}+\frac{8}{3} N_{\mathrm{sv}} \frac{b}{b+1}\left(b+\frac{d}{h}\right)\right]$ & $\Phi_{\mathrm{p}}=\frac{\Phi_{\mathrm{v}}}{b+1}\left[1-\frac{2 d}{3 h}+\frac{16}{3} N_{\mathrm{sv}} \frac{b}{b+1}\left(b+\frac{2 d}{h}\right)\right]$ \\
Case 2 & $h s / h \ll 1 \quad$ and $b=0$ & $\Phi_{\mathrm{v}}=1 /\left(1-\frac{h_{\mathrm{s}}}{3 h}+\frac{16}{15} N_{\mathrm{sv}} \frac{h_{\mathrm{s}}^{2}}{h^{2}}\right)$ & $\Phi_{\mathrm{p}}=\Phi_{\mathrm{v}}\left(1-\frac{2 h_{\mathrm{s}}}{3 h}+\frac{28}{5} N_{\mathrm{sv}} \frac{h_{\mathrm{s}}^{2}}{h^{2}}\right)$ \\
Case 3 & $d / h \gg 1$ & $\Phi_{\mathrm{v}}=\frac{h_{\mathrm{s}}}{2 h} /\left[\ln (d / h)-1-8 N_{\mathrm{sv}}+\frac{4 N_{\mathrm{sv}} h_{\mathrm{s}}}{3 h}\right]$ & $\Phi_{\mathrm{p}}=\frac{2 h \Phi_{\mathrm{v}}\left(1+\frac{8}{3} N_{\mathrm{sv}} \frac{h_{\mathrm{s}}}{h}\right)}{h_{\mathrm{s}}}(1)$ \\
Case 4 & $\begin{array}{l}\text { Without Marangoni } \\
\text { terms and } N_{\mathrm{sv}} \ll 1\end{array}$ & $\Phi_{\mathrm{v}}=\frac{3}{8 N_{\mathrm{sv}}}$ & $\Phi_{\mathrm{p}}=2$ \\
Case 5 & $\mathrm{~N}_{\mathrm{sv}} \gg 1$ & $\Phi_{\mathrm{v}}=\frac{2 N_{\mathrm{sv}}}{2 N_{\mathrm{sv}}-1}$ & $\Phi_{\mathrm{p}}=1-\frac{1}{2 N_{\mathrm{sv}}}\left(\frac{\pi^{2}}{6}-1\right)$ \\
\hline
\end{tabular}

To illustrate this conclusion we derived several asymptotic expressions for the mobility functions (see Table 1). The first four cases correspond to small effect of the surface viscosity. If $d / h \ll 1$ (case 1) the surface viscosity parameter, $N_{\mathrm{sv}}$, in $\Phi_{\mathrm{v}}$ and $\Phi_{\mathrm{p}}$ is multiplied by the bulk diffusion parameter $b$. Therefore, if the bulk diffusivity is large enough to suppress the surface tension gradient (i.e. the Marangoni effect), the influence of the surface viscosity will be more pronounced. In case 2 (insoluble surfactants and large interfacial mobility) the bulk diffusivity parameter, $b$, is zero. In this case $N_{\mathrm{sv}}$ is multiplied by $h_{\mathrm{s}}^{2} / h^{2}$ in $\Phi_{\mathrm{v}}$ and $\Phi_{\mathrm{p}}$ (see Table 1). As a result, for insoluble surfactant the Gibbs elasticity leads to more pronounced effect of immobilization of the interface and the effect of surface viscosity is consequently small. In the case of large mobility of the interfaces, i.e. with $d / h \gg 1$ (case 3 in Table 1), the surface viscosity affects the drainage velocity and the formation of dimple or pimple significantly. This is well visible when the Marangoni effect is negligible (case 4 in Table 1). Then the drainage velocity depends only on the surface viscosity and the inversion thickness can achieve larger values than for tangentially immobile interfaces.
In order to illustrate these effects we calculated the mobility functions for a wide range of values of the mobility parameter, $d / h$, with three values of the bulk diffusivity parameter $(b=0,1$, and 10$)$ and with surface viscosity parameter $N_{\text {sv }}=0.1$. The numerical results are presented in Fig. 4 with solid lines. The results calculated from the respective asymptotic expressions are plotted with dashed lines. Obviously, the exact and the asymptotic formulae for the mobility functions agree well. The general trend of the increase of the drainage velocity with increasing mobility of the interfaces is demonstrated in Fig. 4 (a). In Fig. 4 (b) the increase of the mobility function for the pressure when the mobility of the interfaces increases is shown-this effect favors the dimple formation and hinders the pimple formation.

In order to demonstrate the influence of surfactants on the inversion and pimple thickness we solved numerically the respective Eqs. (25a, b) with van der Waals attraction forces. The results are presented in Figs. 5 and 6. When the surface viscosity effect is neglected $\left(N_{\mathrm{sv}}=0\right)$, the mobility functions, $\Phi_{\mathrm{v}}$ and $\Phi_{\mathrm{p}}$, depend only on the length parameter, $d$, and the thickness, $h$. The numerical results for different values of the Hamaker constant $\left(A_{\mathrm{H}}=0, A_{\mathrm{H}}=4 \times 10^{-21} \mathrm{~J}\right.$ and $A_{\mathrm{H}}=4 \times$ 
$10^{-20} \mathrm{~J}$ ) are plotted in Fig. 5. Both quantities, $d$ and $h_{\mathrm{it}}$, are normalized by $F /(2 \pi \sigma)$. The bubbles radius and the surface tension are chosen to be $R_{\mathrm{c}}=20 \mu \mathrm{m}$ and $\sigma=15 \mathrm{mN} \mathrm{m}^{-1}$. The observed general trend is that when the parameter $d$ increases (increasing surface mobility), the inversion thickness decreases. This effect has the following simple explanation: when the surfaces are with lower Gibbs elasticity (i.e. more mobile) the hydrodynamic friction is lower, so that the gap width, at which the viscous stress can counterbalance the capillary pressure and provoke dimple formation, is smaller. When the disjoining pressure, $\Pi$, is zero, the solution for the inversion thickness exists for the whole range of the parameter $d$. If the attractive van der Waals disjoining pressure is taken into account, above a certain surface mobility (above a given value of the parameter $d$, which depends also on the Hamaker constant, $A_{\mathrm{H}}$ ) no solution for the inver-
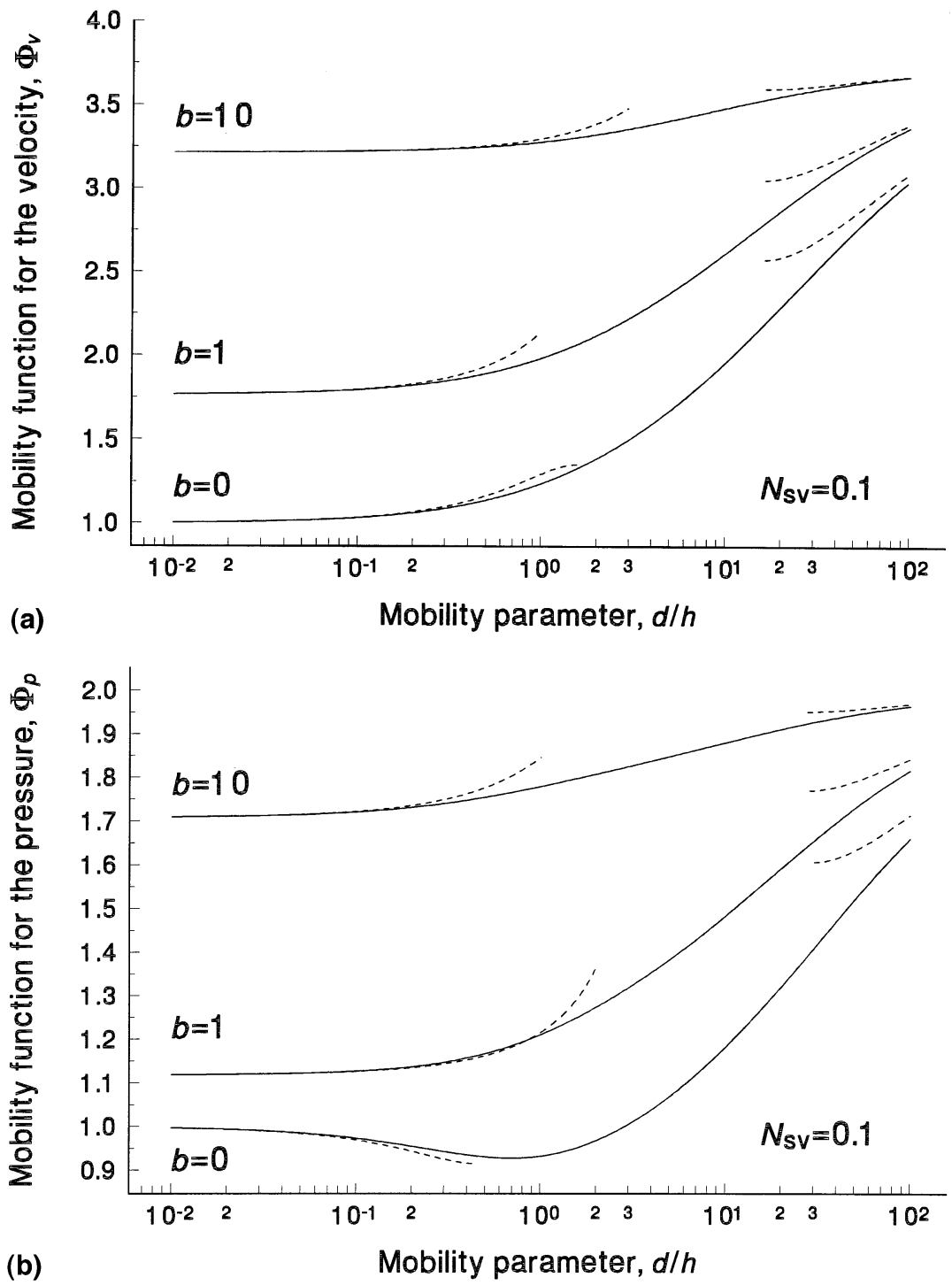

Fig. 4. Dependence of the mobility functions on the dimensionless mobility parameter at $N_{\text {sv }}=0.1$ and different values of $b$ : 0 (insoluble surfactant), 1, and 10. (a) Mobility function for the velocity, $\Phi_{\mathrm{v}}$; (b) mobility function for the pressure, $\Phi_{\mathrm{p}}$. 


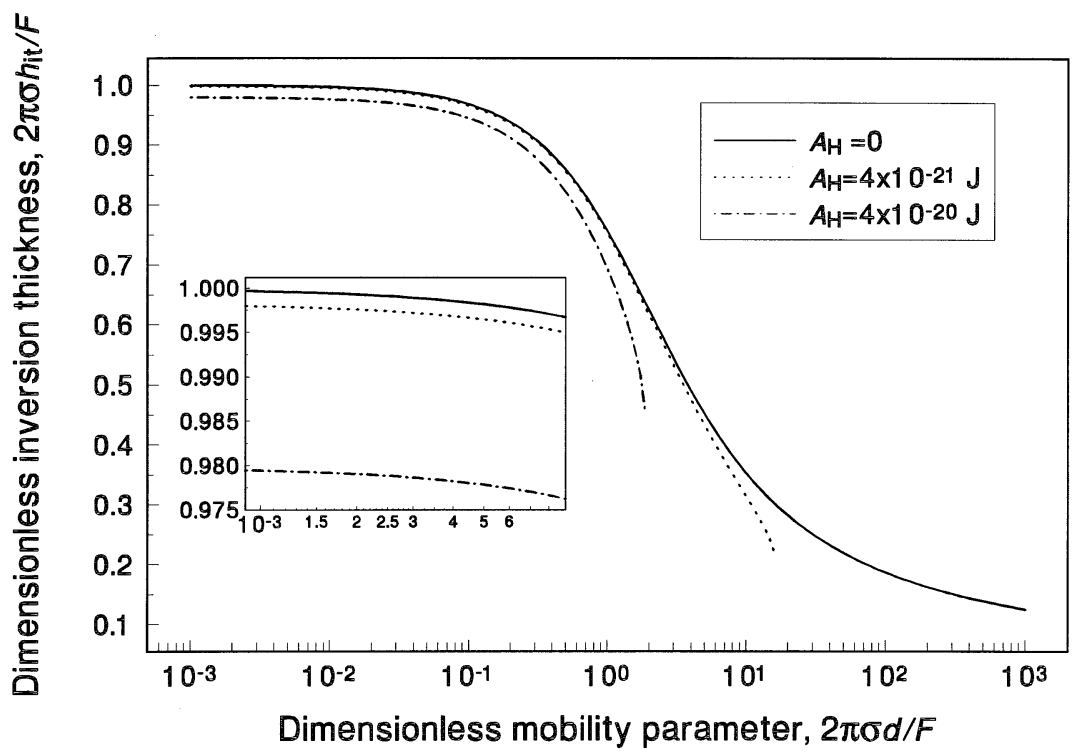

Fig. 5. Inversion thickness, $2 \pi \sigma h_{\mathrm{it}} / F$, vs. parameter $2 \pi \sigma d / F$ in the case of $N_{\mathrm{sv}}=0$, for $A_{\mathrm{H}}=0, A_{\mathrm{H}}=4 \times 10^{-21} \mathrm{~J}$, and $A_{\mathrm{H}}=4 \times$ $10^{-20} \mathrm{~J}$. The drop radius and the interfacial tension are $R_{\mathrm{c}}=20 \mu \mathrm{m}$ and $\sigma=15 \mathrm{mN} \mathrm{m}^{-1}$, respectively.

sion thickness exists, i.e. no dimple can be formed. The bigger the attractive force, the lower the inversion thickness and more difficult the dimple formation is.

The inversion and pimple thicknesses as functions of the surface mobility for two different values of the Hamaker constants, $A_{\mathrm{H}}$, are compared in Fig. 6. The other parameters are the same as in Fig. 5. The vertical lines correspond to the values of the dimensionless mobility parameter, $d$, at which no solution for the inversion thickness exists - above this value only pimple can appear. One sees, that with larger attractive forces the pimple appears at lower surface mobility.

If the Marangoni effect is negligible and the surface viscosity is large enough, i.e. if $N_{\mathrm{sv}} h_{\mathrm{s}} / h \gg 1$ and $N_{\mathrm{sv}} b \gg 1, N_{\mathrm{sv}}$ in the right hand side of Eq. (23) will be a large parameter and the solution can be written as series in terms of $1 / N_{\mathrm{sv}}$. The leading order term of the solution for $\tilde{U}_{0}$ is

$\tilde{U}_{0}=\frac{\tilde{V}_{0}}{8 N_{\mathrm{sv}}} \frac{\ln \tilde{H}_{0}}{x}$

i.e. the surface velocity is inversely proportional to $N_{\mathrm{sv}}$. Upon substitution of Eq. (26) into Eq. (22) and integration of the result for the pressure gradient, the asymptotic expressions for the mobility functions $\Phi_{\mathrm{v}}$ and $\Phi_{\mathrm{p}}$ can be derived. The final results for the mobility parameters are given in Table 1 (case 5). Since the surface viscosity hinders the interfacial mobility, the surface velocity, the inversion and the pimple thicknesses reach eventually their values for tangentially immobile interfaces as $N_{\text {sv }}$ grows (see Section 3).

\section{Numerical results and discussion of the general case}

The exact solution (within leading order terms with respect to $\alpha$ but with no other approximations) is described in Appendix B. Therein expressions for the mobility functions, $\Phi_{\mathrm{v}}$ and $\Phi_{\mathrm{p}}$, are derived [see Eqs. (B3) and (B5)]. We used them in the transcendental Eqs. (25a, b) for the calculation of the inversion and pimple thicknesses. It is important to note that, just as it is in the approximate case, considered above in Section 4, the surface viscosity parameter in all coefficients of Eq. (B1) appears as the combinations $N_{\mathrm{sv}} h_{\mathrm{s}} / h=$ $\eta_{\mathrm{s}} D_{\mathrm{s}} /\left(h E_{\mathrm{G}} R_{\mathrm{c}}\right)$ and $N_{\mathrm{sv}} b=\eta_{\mathrm{s}} D /\left(h_{\mathrm{a}} E_{\mathrm{G}} R_{\mathrm{c}}\right)$. Therefore, in the general case too, the surface viscosity 
can influence the drainage and film formation only when either the Gibbs elasticity or the bubble size are small. The effect is more pronounced with thinner films. The numerical results for the mobility function, $\Phi_{\mathrm{v}}$, for $N_{\mathrm{sv}}=1$ are given in Fig. 7 and for $N_{\mathrm{sv}}=0.1$ in Fig. 4. As one should expect, the increase of the surface viscosity number from 0.1 (Fig. 4) to 1.0 (Fig. 7) decreases the mobility of the interfaces.

The role of the surface viscosity on the dimple formation is illustrated in Fig. 8 for typical values of the mobility parameter, $d$. The different curves correspond to surface viscosity parameters $N_{\mathrm{sv}}=$ $0.001,0.01,0.1$, and 0.5 . It is evident that when the surface diffusivity is high (large $d$ ), the inversion thickness depends stronger on the surface viscosity. With increasing surface viscosity the inversion thickness increases as well because of the rising hydrodynamic friction. At high surface viscosity (see the curve for $N_{\mathrm{sv}}=0.5$ ) the dimensionless inversion thickness tends to 1, i.e. then the interfaces behave as almost immobile.

Until now our discussion was not concerned with a specific type of surfactant. At the same time, it is well known that the stability (the coalescence or flocculation) of foams and emulsions depend strongly on whether or not thin liquid films form between the colliding bubbles or droplets [28] and the formation of such films depends in terms on the surfactant properties and concentration. That is why we calculated, using the full Eqs. $(25 \mathrm{a}, \mathrm{b})$ and Appendix B, the inversion and pimple thickness as functions of the surfactant concentration for three different kind of surfactants: nonionic (Triton X-100), ionic (sodium dodecyl sulfate, SDS) and protein isolates (BSA) in the continuous phase (see Figs. 9 and 10). We assume that the electrostatic repulsion was suppressed by adding $0.1 \mathrm{M} \mathrm{NaCl}$ to the SDS solution. Although BSA is not a typical surfactant, it was found that all three obey Langmuir adsorption isotherm with the parameters presented in Table $2[29,30]$. The equilibrium surfactant adsorption, $\Gamma_{0}$, and the interfacial tension, $\sigma$, are related with the surfactant concentration, $c_{0}$, by Langmuir-Szyszkowski adsorption isotherm:

$$
\frac{\Gamma_{0}}{\Gamma_{\infty}}=\frac{c_{0}}{B+c_{0}}, \quad \sigma=\sigma_{\mathrm{p}}+k_{\mathrm{B}} T \Gamma_{\infty} \ln \left(1-\frac{\Gamma_{0}}{\Gamma_{\infty}}\right)
$$

where $T$ is the temperature, $k_{\mathrm{B}}$ is the Boltzmann constant, $\sigma_{\mathrm{p}}$ is the interfacial tension of the pure solvent, $\Gamma_{\infty}$ is the saturation adsorption, and $B$ is

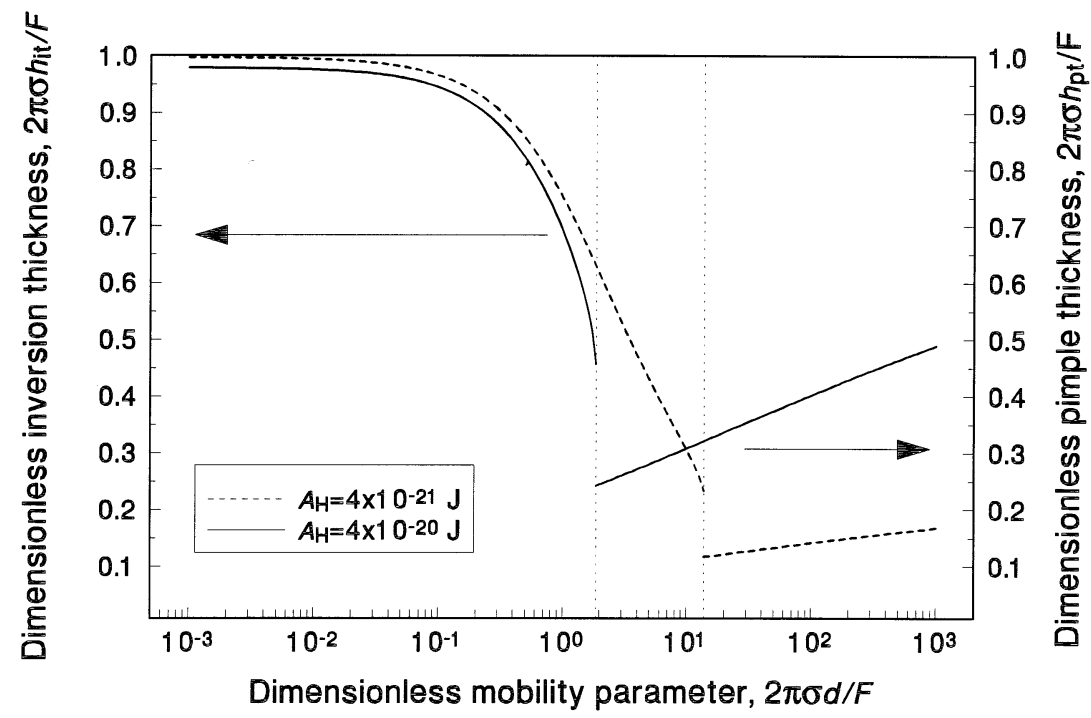

Fig. 6. Inversion and pimple thicknesses vs. surface mobility parameter at different values of the attractive force. The other system parameters are the same as in Fig. 5. 


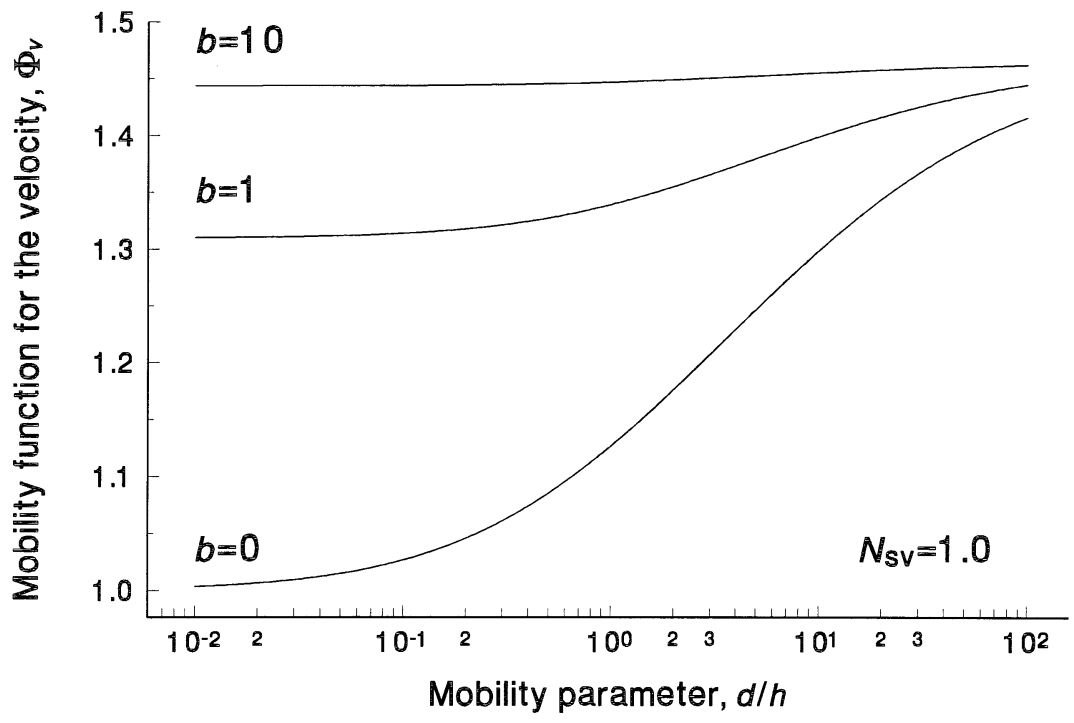

Fig. 7. Dependence of the mobility function, $\Phi_{\mathrm{v}}$, on the dimensionless mobility parameter at $N_{\mathrm{sv}}=1$ and different values of $b$ : 0 (insoluble surfactant), 1, and 10.

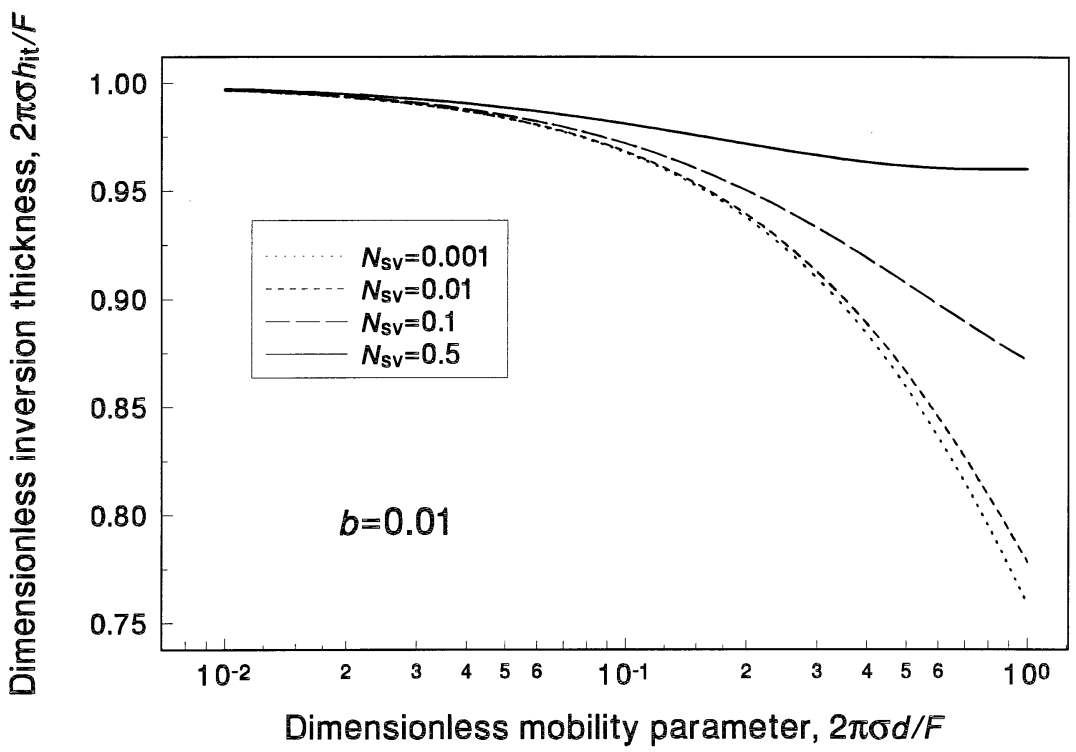

Fig. 8. Influence of the mobility parameter, $2 \pi \sigma d / F$, on the thickness of film formation, $h_{\mathrm{it}}$, for different values of surface viscosity number $N_{\mathrm{sv}}=0.001,0.01,0.1$, and 0.5 , and bulk diffusion parameter $b=0.01$.

the adsorption constant, which is related with the specific energy of adsorption. The corresponding relationships for $b$ and $h_{\mathrm{s}}$ read

$$
b=\frac{3 \eta D}{k_{\mathrm{B}} T \Gamma_{0}} \frac{B}{\Gamma_{\infty}}\left(1-\frac{\Gamma_{0}}{\Gamma_{\infty}}\right)^{-1},
$$



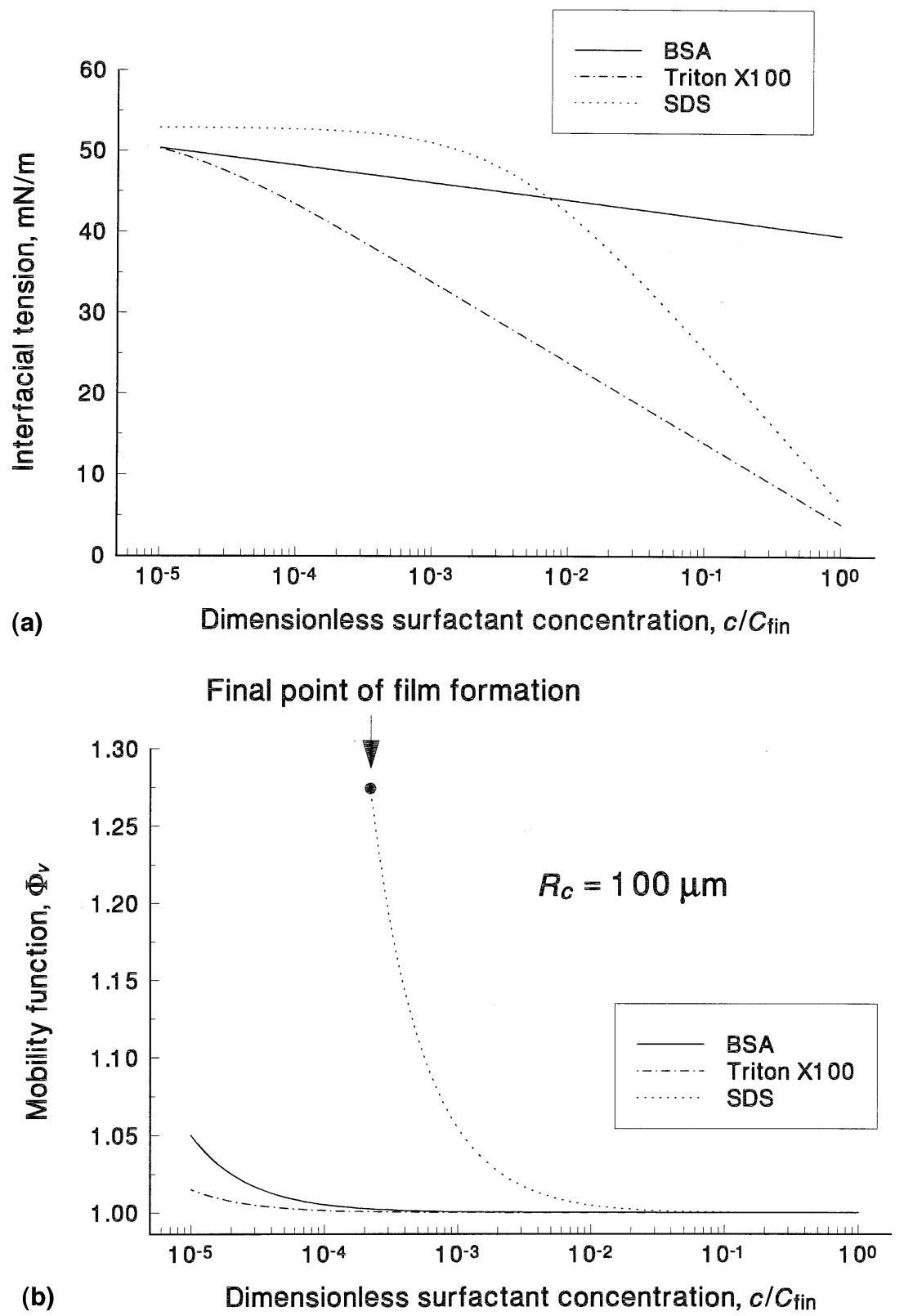

Fig. 9. Dependence of the inversion thickness on the surfactant concentration for bovine serum albumin (BSA) (solid line), Triton X-100 (dashed line) and sodium dodecyl sulphate (SDS) + 0.1 M NaCl: (a) interfacial tension; (b) mobility function, $\Phi_{\mathrm{v}}$; (c) inversion thickness, $h_{\mathrm{it}}$.

$h_{s}=\frac{6 \eta D_{\mathrm{s}}}{k_{\mathrm{B}} T \Gamma_{0}}\left(1-\frac{\Gamma_{0}}{\Gamma_{\infty}}\right)$

The calculated dependence of the interfacial tensions of the three solutions at the interface water solution-dodecane vs. the dimensionless surfactant concentration, $c / C_{\text {fin }}$, is shown in Fig. 9 (a) (the concentration, $C_{\text {fin }}$, corresponds to $\mathrm{CMC}$ for Triton X-100 and SDS and to the final concentration of validity of the Langmuir isotherm 


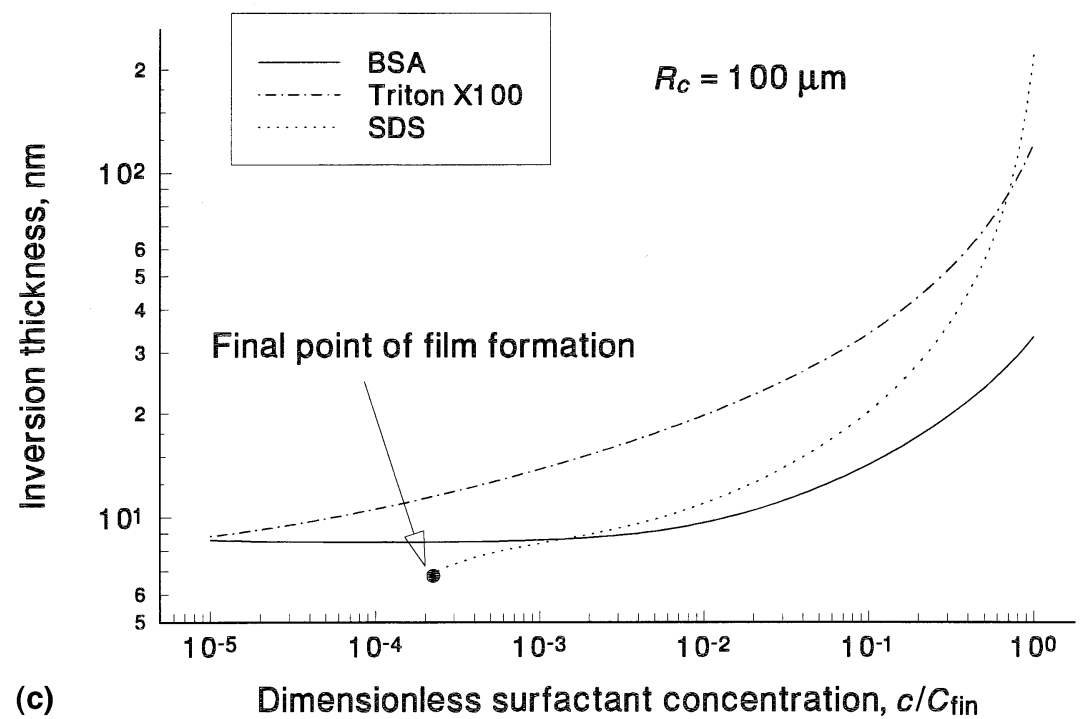

Fig. 9. (Continued)

For BSA[29,30]). Because of the lack of data, we assume for the surface viscosity a linear dependence on the adsorption: $\eta_{\mathrm{s}}=\eta_{\mathrm{s}, \max } \Gamma_{0} / \Gamma_{\infty}$. Since we could not find the experimental data for the surface diffusion coefficient, $D_{\mathrm{s}}$, it was taken equal to the bulk diffusion coefficient, $D$. When calculating the van der Waals attractive force instead of the Hamaker constant we used the effective interaction parameter, $A_{\text {eff }}$, defined by Eqs. (5.9.1-5) in Refs. [21], which takes into account the electromagnetic retardation. The driving force, $F$, was taken to be the buoyancy force for dodecane drops.

Since for a relatively large drop radius $R_{\mathrm{c}}=100$ $\mu \mathrm{m}$ the solution for the inversion thickness exists, a pimple cannot form. The mobility functions, $\Phi_{\mathrm{v}}$, and the inversion thicknesses as functions of the concentration are plotted for this case in Fig. 9 $(b, c)$. As one should expect, at high surfactant concentrations the mobility functions, $\Phi_{\mathrm{v}}$, tend to unity [Fig. 9 (b)], i.e. the interfaces become immobile, but this happens for SDS at concentrations, which are by two orders of magnitude larger than for BSA and Triton X-100. The significant increase of the inversion thickness, observed in Fig. 9 (c) at high surfactant concentrations for all three surfactants, results from the respective de- crease of the interfacial tension. Similarly, the inversion thickness for BSA is lower than for Triton X-100 and SDS, because its interfacial tension is higher [Fig. 9 (a)]. For better clarity of the presentation we have not shown in Fig. 9 (c) the curves for the inversion thickness for tangentially immobile interfaces, but the reader could realize that they should coincide with the respective curves in Fig. 9 (c) for the concentration range where the respective mobility functions in Fig. 9 (b) are approximately equal to 1, i.e. where the interfaces are tangentially immobile. However, since the interfacial mobility with SDS is much higher [Fig. 9 (b)], the deviation of the inversion thickness from its value for an immobile surface starts at higher surfactant concentrations. Below a given concentration of SDS [below the point, marked in Fig. 9 (c)]. Eq. (25a) for the inversion thickness has no solution, i.e. below this point a dimple cannot form.

As shown in Section 3, below a given bubble radius only pimple can form. The results for the mobility function, $\Phi_{\mathrm{v}}$, and the pimple thickness, $h_{\mathrm{pt}}$, for small droplets, of radius $R_{\mathrm{c}}=20 \mu \mathrm{m}$ (they form only pimples) are plotted in Fig. 10 (a, b). It is important to remember that the value of the pimple thickness depends on the mobility of the 


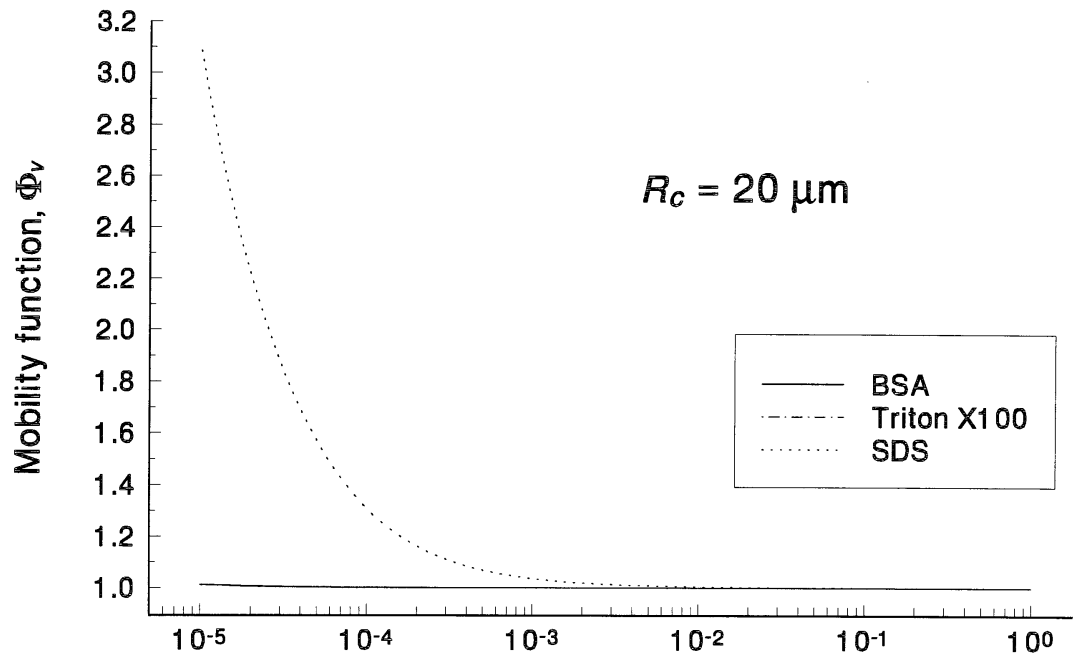

(a) Dimensionless surfactant concentration, $c / C_{\text {fin }}$

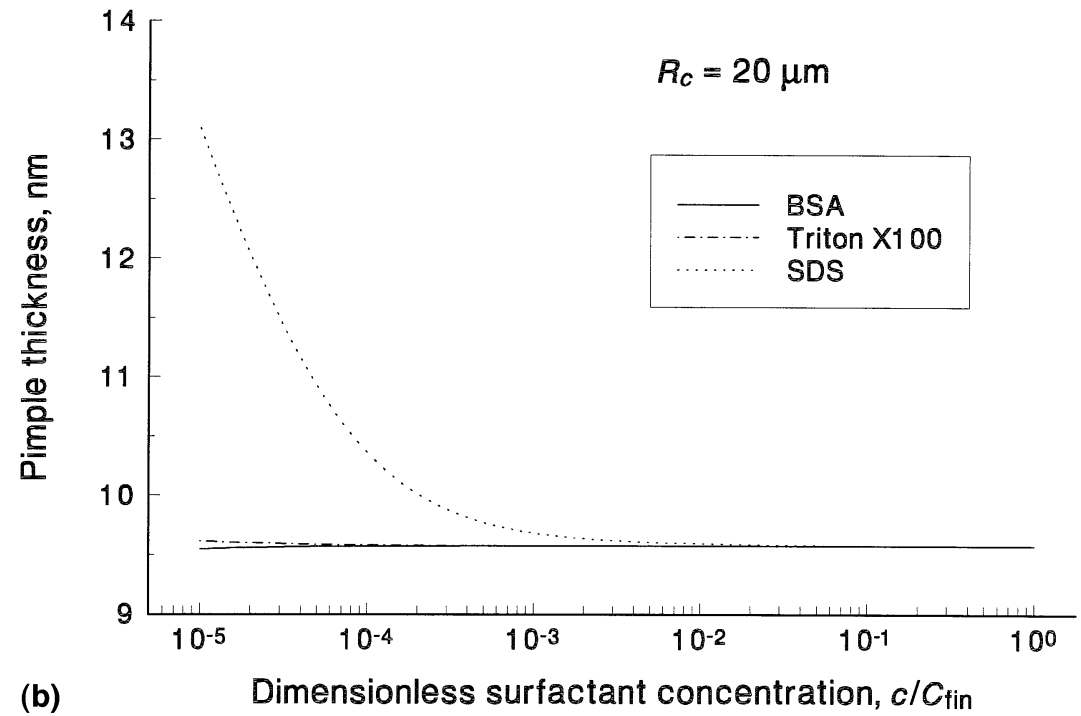

Fig. 10. Dependence of the pimple thickness on the surfactant concentration for bovine serum albumin (BSA) (solid line), Triton X-100 (dashed line) and sodium dodecyl sulphate (SDS) $+0.1 \mathrm{M} \mathrm{NaCl}$ : (a) mobility function, $\Phi_{\mathrm{v}}$; (b) pimple thickness, $h_{\mathrm{pt}}$.

interface and the magnitude of attractive forces but not on the interfacial tension. The observed significant increase of the mobility function and the pimple thickness in the case of SDS in Fig. 10 $(a, b)$ result from the higher mobility of the interface with SDS compared to that with Triton X-100 or BSA - for latter two surfactants the mobility function at all concentrations is nearly 1 and the pimple thickness is approximately constant.

\section{Conclusions}

A theoretical model for calculation of the influence of surfactants on the drainage velocity, inversion and pimple thickness of two slightly deformed bubbles is developed. It takes into account the influence of Gibbs elasticity, bulk and surface diffusion and surface viscosity, which are included in the characteristic parameters, see Eq. (3). The governing equations [Eqs. (8a-d)] 
derived from the lubrication approximation for small shape perturbation are used for numerical calculations in order to find the inversion and pimple thicknesses. Analytical expressions, valid for small and large effect of the surface viscosity or tangentially immobile interfaces are derived [see Eqs. (16, 19, 24, 25a, b) and Table 1]. They can serve for simple estimations of the effect of the surfactant.

The quantitative calculations (see Sections 3-5) show that with increasing surface mobility, surface tension and the attractive disjoining pressure (e.g. van der Waals attraction) the inversion thickness, at which a film forms, decreases. In contrast, larger driving force, Gibbs elasticity and surface viscosity favor film formation. All factors whichincrease the hydrodynamic resistance in the gap region lead to earlier film formation. The attractive disjoining pressure hinders the film formation and if its value is large enough it can prevent the film formation altogether. In this case at a given thickness the curvature of the droplets at the gap increases and a pimple is formed. The pimple formation thickness does not depend on the interfacial tension. The pimple thickness becomes larger with larger surface mobility and attractive intermolecular force, and it is smaller for larger driving force. The pimple thickness can be interpreted as a special kind of instability which is provoked only by the disjoining pressure, not by mechanical and/or thermal fluctuations. The problem of fluctuational stability and the critical thickness of rupture of the intervening film is considered in Ref. [19].

\section{Acknowledgements}

This work was supported by Inco-Copernicus project No. PL979098.

\section{Appendix A. Solution of Eqs. $(8 a-d)$ in the case of small effect of the surface viscosity}

In the common case of small effect of the surface viscosity, i.e. when $\mathrm{N}_{\mathrm{sv}} \ll 1$, the right hand side of Eq. (23) is small. Hence the leading order term for the surface velocity is

$$
\tilde{U}_{0}=\frac{x \tilde{V}_{0}}{2}\left[\frac{1}{\tilde{H}_{0}}-\frac{1}{(b+1) \tilde{H}_{0}+\tilde{h}_{\mathrm{s}}}\right]+O\left(N_{\mathrm{sv}}\right)
$$

where the dimensionless surface diffusion parameter, $\tilde{h_{\mathrm{s}}}$, is defined as $\tilde{h_{\mathrm{s}}} \equiv h_{\mathrm{s}} / h$. Substitution of Eq. (A1) into Eq. (22) yields:

$$
\begin{aligned}
\frac{\partial \tilde{P}_{0}}{\partial x}= & -\frac{x \tilde{V}_{0}}{2 \tilde{H}_{0}^{2}\left[(b+1) \tilde{H}_{0}+\tilde{h}_{\mathrm{s}}\right]} \\
& +N_{\mathrm{sv}} \tilde{V}_{0}\left[\frac{1}{\tilde{H}_{0}}-\frac{1}{(b+1) \tilde{H}_{0}+\tilde{h}_{\mathrm{s}}}\right] \\
& \times \frac{\partial}{\partial x}\left[\frac{1}{x} \frac{\partial}{\partial x}\left(x \tilde{U}_{0}\right)\right]+O\left(N_{\mathrm{sv}}^{2}\right)
\end{aligned}
$$

After simple integration the respective expression for the force Eq. (8d) can be represented in

the form:
$1-8 \tilde{W}_{0}=-8 \int_{0}^{\infty} x^{2} \frac{\partial \tilde{P}_{0}}{\partial x} d x$

If we multiply Eq. (A2) by $-8 x^{2}$ and integrate the result from 0 to infinity the final expression for the dimensionless velocity of droplet approach reads

$\tilde{V}_{0}=\left(1-8 \tilde{W}_{0}\right) \Phi_{\mathrm{v}}$

where the mobility function, $\Phi_{\mathrm{v}}$, was found to be

$$
\begin{aligned}
\Phi_{\mathrm{v}}^{-1}= & \frac{2}{\tilde{d}(b+1)}\left[\frac{\tilde{d}+1}{\tilde{d}} \ln (\tilde{d}+1)-1\right] \\
& +\frac{8 N_{\mathrm{sv}}}{3}\left[1+\frac{1}{(b+1)^{2}(\tilde{d}+1)}\right]+ \\
& +16 N_{\mathrm{sv}} \frac{\tilde{d}+1}{\tilde{d}^{2}(b+1)}\left[\frac{2}{\tilde{d}} \ln (\tilde{d}+1)-\frac{\tilde{d}+2}{\tilde{d}+1}\right]
\end{aligned}
$$

In Eq. (A5) the dimensionless mobility parameter, $\tilde{d}$, is defined as $\tilde{d} \equiv \tilde{h}_{\mathrm{s}} /(b+1)$. By setting $N_{\mathrm{sv}}=0$ in Eq. (A5), the respective result from Ref. [10] is recovered. Simple expressions for $\Phi_{\mathrm{v}}$ for several particular cases are given in Table 1.

Direct integration of Eq. (A2) using Eq. (A4) from 0 to infinity gives the following result for the pressure in the gap: $8 \tilde{P}_{0}(0)=\left(1-8 \tilde{W}_{0}\right) \Phi_{\mathrm{p}}$, where the mobility function of the pressure, $\Phi_{\mathrm{p}}$, is

$\Phi_{\mathrm{p}}=\frac{2 \Phi_{\mathrm{v}}}{\tilde{d}(b+1)}\left[1-\frac{1}{\tilde{d}} \ln (\tilde{d}+1)\right]$ 
Table 2

Parameters of the bovine serum albumin (BSA), Triton X-100 and sodium dodecyl sulphate (SDS) $+0.1 \mathrm{M} \mathrm{NaCl}[29,30]^{\mathrm{a}}$

\begin{tabular}{lccc}
\hline & Triton X-100 & SDS $+0.1 \mathrm{M} \mathrm{NaCl}$ & BSA \\
\hline$\Gamma_{\infty}$ & $1.75 \times 10^{-6} \mathrm{~mol} \mathrm{~m}^{-2}$ & $3.75 \times 10^{-6} \mathrm{~mol} \mathrm{~m}^{-2}$ & $0.38 \times 10^{-6} \mathrm{~mol} \mathrm{~m}^{-2}$ \\
$B$ & $4.9 \times 10^{-6} \mathrm{~kg} \mathrm{~m}^{-3}$ & $1.4 \times 10^{-3} \mathrm{~kg} \mathrm{~m}^{-3}$ & $0.124 \times 10^{-6} \mathrm{~kg} \mathrm{~m}^{-3}$ \\
$D$ & $2.6 \times 10^{-10} \mathrm{~m}^{2} \mathrm{~s}^{-1}$ & $6.0 \times 10^{-10} \mathrm{~m}^{2} \mathrm{~s}^{-1}$ & $1.0 \times 10^{-10} \mathrm{~m}^{2} \mathrm{~s}^{-1}$ \\
$C_{\text {fin }}$ & $0.4 \mathrm{~kg} \mathrm{~m}^{-3}(\mathrm{CMC})$ & $0.33 \mathrm{~kg} \mathrm{~m}^{-3}(\mathrm{CMC})$ & $0.6 \mathrm{~kg} \mathrm{~m}^{-3}$ \\
$\eta_{s, \max }$ & $1.0 \times 10^{-6} \mathrm{mPa} \mathrm{s}$ & $1.0 \times 10^{-6} \mathrm{mPa} \mathrm{s}$ & $5.0 \times 10^{-3} \mathrm{mPa} \mathrm{s}$ \\
\hline
\end{tabular}

${ }^{a} \mathrm{CMC}$, critical micelle concentration.

$$
\begin{aligned}
& +\frac{16 \Phi_{\mathrm{v}} N_{\mathrm{sv}}}{3}\left[1+\frac{1}{(b+1)^{2}(\tilde{d}+1)}\right] \\
& -\frac{8 \Phi_{\mathrm{v}} N_{\mathrm{sv}}}{\tilde{d}(b+1)}\left[\frac{\tilde{d}}{\tilde{d}+1}-\frac{4}{\tilde{d}}+\frac{2(\tilde{d}+2)}{\tilde{d}^{2}} \ln (\tilde{d}+1)\right]
\end{aligned}
$$

Simple asymptotic expressions for specific cases are given in Table 1.

\section{Appendix B. General form of the mobility functions}

We presented above (see Sections 3 and 4) asymptotic solutions of Eqs. (22) and (23) for several specific cases. It is not possible however to obtain simple analytic solution of these equations without further approximations. That is why we represented the general solution of Eqs. (22) and (23) for the mobility functions, $\Phi_{\mathrm{v}}$ and $\Phi_{\mathrm{p}}$, as infinite series, which were further treated numerically. The procedure is outlined below.

The boundary conditions of Eq. (23) are: vanishing velocity, $\tilde{U}_{0}$, at the film origin, $x=0$ and at $x \rightarrow \infty$ (far away from the gap region). The wide range of $x$ from zero to infinity makes the numerical solution with a high precision impossible. To overcome this difficulty, we introduce a new variable, $x=\tan (\theta / 2)$, which transforms the infinite range of $x$ values to the finite interval $[0, \pi]$, where the infinity point is transformed to $\theta=\pi$, and the zero point to $\theta=0$. Since with the new variable the approximate unperturbed film profile is $\widetilde{H}_{0}=1 / \cos ^{2}(\theta / 2)$, Eq. (23) acquires the form

$B_{2} N_{\mathrm{sv}} \frac{\partial^{2} \tilde{U}_{0}}{\partial \theta^{2}}+B_{1} N_{\mathrm{sv}} \frac{\partial \tilde{U}_{0}}{\partial \theta}+B_{0} \tilde{U}_{0}=L \tilde{V}_{0}$

where the coefficients are:

$$
\begin{aligned}
B_{2}= & -\tilde{h_{\mathrm{s}}} \cos (3 \theta)-2\left(2 b+\tilde{h_{\mathrm{s}}}\right) \cos (2 \theta)+\tilde{h_{\mathrm{s}}} \cos \theta \\
& +2\left(2 b+\tilde{h_{\mathrm{s}}}\right) \\
B_{1}= & \tilde{h_{\mathrm{s}}} \sin (3 \theta)+2\left(2 b+\tilde{h_{\mathrm{s}}}\right) \sin (2 \theta)+\tilde{h_{\mathrm{s}}} \sin \theta \\
B_{0}= & \tilde{h_{\mathrm{s}}} \cos (2 \theta)-4\left(N_{\mathrm{sv}} \tilde{h_{\mathrm{s}}}-1-b\right) \cos \theta-\tilde{h_{\mathrm{s}}} \\
& -4(1+b)-4 N_{\mathrm{sv}}\left(2 b+\tilde{h_{\mathrm{s}}}\right) \\
L= & \frac{\tilde{h}_{\mathrm{s}}}{8} \sin (3 \theta)+\frac{b}{2} \sin (2 \theta)-\left(b+\frac{3 \tilde{h_{\mathrm{s}}}}{8}\right) \sin \theta
\end{aligned}
$$

Because of the boundary conditions at $\theta=0$ and $\theta=\pi$ and the periodicity of $B_{2}, B_{1}, B_{0}$ and $L$, the solution can be presented as a Fourier series:

$\tilde{U}_{0}=\frac{\tilde{V}_{0}}{8} \sum_{k=1}^{\infty} a_{k} \sin (k \theta)$

where the coefficients $a_{k}$ depend implicitly on the physical parameters. After substitution of the Fourier series into Eq. (B1) we obtain an infinite linear system of equations for the unknown quantities $a_{k}$. This system is of seven-diagonal type and is solved numerically by means of the Thompson algorithm [31]. The special type of the system makes possible the solution of the problem for a large number of coefficients (in our calculations the cut off number was 10000 ).

After substitution of Eq. (B2) into Eq. (22) we obtain the expression for the gradient of the dynamic pressure. If the result is substituted in the force balance [Eq. (A3)], the mobility function for the velocity, $\Phi_{v}$, takes the form:

$\Phi_{\mathrm{v}}=\frac{1}{1-a_{\text {odd }}+a_{\text {ev }}}$

where $a_{\mathrm{ev}}$ and $a_{\mathrm{odd}}$ are the respective sums of the even and odd parts of coefficients $a_{\mathrm{k}}$ :

$a_{\text {odd }}=\sum_{k=0}^{\infty} \frac{a_{2 k+1}}{2(2 k+1)}, \quad a_{\mathrm{ev}}=\sum_{k=1}^{\infty} \frac{k a_{2 k}}{4 k^{2}-1}$ 
A direct integration of the expression for the dynamic pressure gradient from 0 to infinity gives a simple expression for the mobility function of the pressure:

$\Phi_{\mathrm{p}}=\frac{8 \tilde{P}_{0}(0)}{1-\tilde{W}_{0}}=\frac{1-a_{\mathrm{odd}}-a_{\mathrm{ev}}}{1-a_{\mathrm{odd}}+a_{\mathrm{ev}}}$

The mobility parameters, $\Phi_{\mathrm{v}}$ and $\Phi_{\mathrm{p}}$, are complex functions of the thickness, $h$, and the material properties of the interfaces through the parameters $b, h_{\mathrm{s}}$ and $N_{\mathrm{sv}}$, on which the coefficients $a_{k}$ depend.

\section{References}

[1] K.D. Danov, N.D. Denkov, D.N. Petsev, R. Borwankar, Langmuir 9 (1993) 1731.

[2] K.D. Danov, D.N. Petsev, N.D. Denkov, R. Borwankar, J. Chem. Phys. 99 (1993) 7179.

[3] D.N. Petsev, N.D. Denkov, P.A. Kralchevsky, J. Colloid Interface Sci. 176 (1995) 201.

[4] I.B. Ivanov, Pure Appl. Chem. 52 (1980) 1241.

[5] I.B. Ivanov, D.S. Dimitrov, in: I.B. Ivanov (Ed.), Thin Liquid Films, Dekker, New York, 1988, p. 379.

[6] N.D. Denkov, D.N. Petsev, K.D. Danov, J. Colloid Interface Sci. 176 (1995) 189.

[7] P. Becher (Ed.), 1996. Encyclopedia of Emulsion Technology. Dekker, New York.

[8] R.K. Prud'home, S.A. Khan (Eds.), 1996. Foams, Theory, Measurements, and Applications. Dekker, New York.

[9] P. Taylor, Proc. Royal Soc. (Lond.) A 108 (1924) 11.

[10] I.B. Ivanov, D.S. Dimitrov, P. Somasundaran, R.K. Jain, Chem. Eng. Sci. 40 (1985) 137.

[11] R.H. Davis, J.A. Schonberg, J.M. Rallison, Phys. Fluids A1 (1989) 77.

[12] S.G. Yiantsios, R.H. Davis, J. Fluid Mech. 217 (1990) 547.

[13] S.G. Yiantsios, R.H. Davis, J. Colloid Interface Sci. 144
(1991) 412.

[14] K.D. Danov, D.S. Valkovska, I.B. Ivanov. J. Colloid Interface Sci. 211 (1999) 291.

[15] G. Singh, G.J. Hirasaki, C.A. Miller, J. Colloid Interface Sci. 184 (1996) 92.

[16] L. Lobo, I.B. Ivanov, D. Wasan, AIChE J. 39 (1993) 322.

[17] M.A. Delichatsios, R.F. Probstein, J. Colloid Interface Sci. 51 (1975) 394.

[18] I.B. Ivanov, B.P. Radoev, T. Traykov, D.S. Dimitrov, E.D. Manev, Chr. Vassilieff, in: E. Wolfram (Ed.), Proceedings of the International Conference on Colloid and Surface Science, vol. 1, Akademia Kiado, Budapest, 1975, p. 583.

[19] D.S. Valkovska, K.D. Danov, I.B. Ivanov, 1999. Colloid Surfaces A (submitted).

[20] J.N. Israelachvili, Intermolecular and Surface Forces, Academic Press, London, 1992.

[21] W.B. Russel, D.A. Saville, W.R. Schowalter, Colloidal Dispersions, Cambridge University Press, Cambridge, 1989.

[22] B.V. Derjaguin, Theory of Stability of Colloids and Thin Films, Plenum Press, New York, 1989.

[23] I.B. Ivanov, D.S. Dimitrov, Colloid Polym. Sci. 252 (1974) 982.

[24] K.D. Danov, I.B. Ivanov, 1997. In: Proceedings of the Second World Congress on Emusion, vol. 2, Bordeaux, paper no. 2-3-154.

[25] I.B. Ivanov, K.D. Danov, P.A. Kralchevsky, 1998. Colloids and Surfaces 152 (1999) 161.

[26] Z. Zapryanov, A.K. Malhotra, N. Aderangi, D.T. Wasan, Int. J. Multiphase Flow 9 (1983) 105.

[27] A.K. Malhotra, D.T. Wasan, Chem. Eng. Commun. 55 (1987) 95

[28] I.B. Ivanov, P.A. Kralchevsky, Colloids and Surfaces 128 (1997) 155.

[29] A. Bonfillon, D. Langevin, Langmuir 9 (1993) 2172.

[30] R. Suttiprasit, V. Krisdhasima, J McGuire, J. Colloid Interface Sci. 154 (1992) 316.

[31] W.H. Press, S.A. Teukolsky, W.T. Vetterling, B.P. Flannery, Numerical Recipes in FORTRAN. The Arts of Scientific Computing, Cambridge University Press, Cambridge, 1992.

[32] V. Cristini, J. Blawzdziewicz, M. Loewenberg, J. Fluid Mech. 366 (1998) 259. 